\title{
QUANTUM ELECTRODYNAMICS WITH SELF-CONJUGATED EQUATIONS WITH SPINOR WAVE FUNCTIONS FOR FERMION FIELDS
}

\author{
V. P. Neznamov ${ }^{1,2 *}$, V. E. Shemarulin ${ }^{1 \dagger}$ \\ ${ }^{1}$ Russian Federal Nuclear Center-All-Russian Research Institute of Experimental Physics, \\ Mira pr., 37, Sarov, 607188, Russia \\ ${ }^{2}$ National Research Nuclear University MEPhI, Moscow, 115409, Russia
}

\begin{abstract}
Quantum electrodynamics (QED) with self-conjugated equations with spinor wave functions for fermion fields is considered. In the low order of the perturbation theory, matrix elements of some of QED physical processes are calculated. The final results coincide with cross-sections calculated in the standard QED. The self-energy of an electron and amplitudes of processes associated with determination of the anomalous magnetic moment of an electron and Lamb shift are calculated. These results agree with the results in the standard QED. Distinctive feature of the developed theory is the fact that only states with positive energies are present in the intermediate virtual states in the calculations of the electron self-energy, anomalous magnetic moment of an electron and Lamb shift. Besides, in equations, masses of particles and antiparticles have the opposite signs.
\end{abstract}

Keywords: Self-conjugated equations with spinor wave functions; quantum electrodynamics; Feynman diagrams; self-energy of an electron; anomalous magnetic moment of an electron; Lamb shift; self-energy function of a photon.

PACS numbers: 03.65.-w, 04.20.-q

\section{Introduction}

In quantum mechanics, motion of half-spin particles is usually described by Dirac equation with first-order derivatives with respect to space-time variables of bispinor wave function [1].

Motion of fermions can also be described by self-conjugated equations with spinor wave functions [2].

Under transformation of the Dirac equation with bispinor wave function to selfconjugated equations with spinor wave functions, the energy of the particle (antiparticle) is preserved while probability densities of particle (antiparticle) are different. This leads to new physical consequences. For instance, in case of black holes, nonregular stationary solutions of Dirac equation in external gravitational and electromagnetic Schwarzschild, Reissner-Nordström, Kerr, Kerr-Newman fields become regular stationary solutions of self-conjugated equations with square-integrable spinor wave functions (see Refs. [3]-[5]).

The analysis of equations with spinor wave functions in the Coulomb repulsive field has shown existence of the impenetrable potential barrier in the effective potential with the radius proportional to the classical fermion radius and inversely proportional to fermion energy (at $\left.E \gg m c^{2}\right)$ ), where $E$ and $m$ are fermion energy and mass [6]. The existence of the impenetrable barrier does not contradict the results of the experiments in probing the internal structure of an electron [7] and

\footnotetext{
*vpneznamov@mail.ru, vpneznamov@vniief.ru

†VEShemarulin@vniief.ru
} 
has no effect on the cross-section of the Coulomb scattering of electrons in the low order of the perturbation theory.

In this paper, we present quantum electrodynamics (QED) with self-conjugated equations with spinor wave functions for fermion fields.

In Sec. 2, we present the self-conjugated equations with spinor wave functions 6. In Sec. 3, we briefly describe the quantum electrodynamics formalism with fermion equations with spinor wave functions. In Sec. 4, we analyze self-energy Feynman diagrams for the QED version under consideration. In Secs. 5, and 6, we calculate matrix elements to determine the anomalous electron moment and Lamb shift by the example of radiative corrections to Coulomb scattering of electrons. In Sec. 7, we discuss the obtained results.

Let us specify these results:

1. In the low order of the perturbation theory, we examine matrix elements of the Coulomb scattering of electrons, scattering of an electron on a proton, Compton effect, and electron-positron pair annihilation. The final results agree with the similar values calculated in the standard QED when using the Dirac equation with the bispinor wave function.

2. The matrix elements for determining the anomalous magnetic moment of an electron and the Lamb shift agree with the matrix elements determined in the standard QED.

3. The self-energy of an electron with ultraviolet logarithmic divergence agrees with the value calculated in the standard QED.

4. Distinctive feature of the developed theory is the fact that only states with positive energies are present in the intermediate virtual states in the calculations of the electron self-energy, anomalous magnetic moment of an electron and Lamb shift. In this case, in the standard QED, the self-energy linearly diverges at the infinite upper limit of integration. Only accounting of the contribution of virtual states with negative energies leads to ultraviolet logarithmic divergence of self-energy (see, for example, Ref. [8]).

5. In equations, masses of particles and antiparticles have the opposite signs.

6. Both for electrons and for positrons, in theory there are no transitions between the states with positive and negative energies. In theory, the concept of creation and annihilation of virtual electron-positron pairs is not necessary.

In App. A, operators of interaction $\sim e^{2}, e^{3}$ are presented. In App. $\mathrm{B}$, the calculation results for some QED effects are presented. In App. C, the contributions of some diagrams to the Lamb shift of atomic levels are presented.

In this paper, we use the system of units of $\hbar=c=1$ and the Minkowski space-time signature.

$$
\eta_{\mu \nu}=\operatorname{diag}[1,-1,-1,-1] .
$$

In (1) $\mu, \nu=0,1,2,3$. 


\section{Self-conjugated equations with spinor wave func- tions for fermions in the external electromag- netic field}

For fermions, with mass $m$ and charge $e$, moving in the external electromagnetic field, the Dirac equation can be written as follows:

$$
\left[p^{0}-e A^{0}(\mathbf{r}, t)-\boldsymbol{\alpha}(\mathbf{p}-e \mathbf{A}(\mathbf{r}, t))-\beta m\right] \psi(\mathbf{r}, t)=0 .
$$

Here, $\psi(\mathbf{r}, t)$ is a bispinor wave function; $A^{0}(\mathbf{r}, t), \mathbf{A}(\mathbf{r}, t)$ are potentials of the electromagnetic field; $\alpha^{k}, \beta$ are four-dimensional Dirac matrices, $k=1,2,3$;

$p^{0}=i(\partial / \partial t), \mathbf{p}=-i \vec{\nabla}$.

Let

$$
\psi(\mathbf{r}, t)=\left(\begin{array}{c}
\varphi(\mathbf{r}, t) \\
\chi(\mathbf{r}, t)
\end{array}\right)
$$

where $\varphi(\mathbf{r}, t), \chi(\mathbf{r}, t)$ are spinor wave functions. Then, the following expressions follow from the equation (2)

$$
\begin{aligned}
& \left(p^{0}-e A^{0}-m\right) \varphi=\boldsymbol{\sigma}(\mathbf{p}-e \mathbf{A}) \chi \\
& \left(p^{0}-e A^{0}+m\right) \chi=\boldsymbol{\sigma}(\mathbf{p}-e \mathbf{A}) \varphi \\
& \chi=\frac{1}{p^{0}-e A^{0}+m} \boldsymbol{\sigma}(\mathbf{p}-e \mathbf{A}) \varphi \\
& \varphi=\frac{1}{p^{0}-e A^{0}-m} \boldsymbol{\sigma}(\mathbf{p}-e \mathbf{A}) \chi
\end{aligned}
$$

Here, $\sigma^{i}$ are Pauli matrices. From the equalities (4) and (5), we can obtain the equations of

$$
\begin{aligned}
& {\left[p^{0}-e A^{0}-m-\boldsymbol{\sigma}(\mathbf{p}-e \mathbf{A}) \frac{1}{p^{0}-e A^{0}+m} \boldsymbol{\sigma}(\mathbf{p}-e \mathbf{A})\right] \varphi=0,} \\
& {\left[p^{0}-e A^{0}+m-\boldsymbol{\sigma}(\mathbf{p}-e \mathbf{A}) \frac{1}{p^{0}-e A^{0}-m} \boldsymbol{\sigma}(\mathbf{p}-e \mathbf{A})\right] \chi=0 .}
\end{aligned}
$$

Equation (6) differs from equation (7) by the replacement of $p^{0} \rightarrow-p^{0}$, $\mathbf{p} \rightarrow-\mathbf{p}, e \rightarrow-e$ or by the replacement of $+m \rightarrow-m$.

If we multiply Eq. (6) on the left by operator $\left(p^{0}-e A^{0}+m\right)$, and Eq. (7) - by operator $\left(p^{0}-e A^{0}-m\right)$, we will obtain that

$$
\begin{aligned}
& {\left[\left(p^{0}-e A^{0}\right)^{2}-m^{2}-\left(p^{0}-e A^{0}+m\right) \boldsymbol{\sigma}(\mathbf{p}-e \mathbf{A})\right.} \\
& \left.\times \frac{1}{p^{0}-e A^{0}+m} \boldsymbol{\sigma}(\mathbf{p}-e \mathbf{A})\right] \varphi=0, \\
& {\left[\left(p^{0}-e A^{0}\right)^{2}-m^{2}-\left(p^{0}-e A^{0}-m\right) \boldsymbol{\sigma}(\mathbf{p}-e \mathbf{A})\right.} \\
& \left.\times \frac{1}{p^{0}-e A^{0}-m} \boldsymbol{\sigma}(\mathbf{p}-e \mathbf{A})\right] \chi=0 .
\end{aligned}
$$

Equations (8) and (9) must be reduced to the self-conjugated form [6]-[10]. It is worth to note that the similarity transformation operator is written in the closed form of

$$
\Phi=g_{\varphi} \varphi, \quad \mathrm{X}=g_{\chi} \chi
$$


where

$$
g_{\varphi}=\left(p^{0}-e A^{0}+m\right)^{-1 / 2}, \quad g_{\chi}=\left(p^{0}-e A^{0}-m\right)^{-1 / 2} .
$$

After the transformations, Eqs. (8) and (9) assume the self-conjugated form of

$$
\begin{aligned}
& {\left[\left(p^{0}-e A^{0}\right)^{2}-m^{2}-\left(p^{0}-e A^{0}+m\right)^{1 / 2} \boldsymbol{\sigma}(\mathbf{p}-e \mathbf{A})\right.} \\
& \left.\times \frac{1}{p^{0}-e A^{0}+m} \boldsymbol{\sigma}(\mathbf{p}-e \mathbf{A})\left(p^{0}-e A^{0}+m\right)^{1 / 2}\right] \Phi=0, \\
& {\left[\left(p^{0}-e A^{0}\right)^{2}-m^{2}-\left(p^{0}-e A^{0}-m\right)^{1 / 2} \boldsymbol{\sigma}(\mathbf{p}-e \mathbf{A})\right.} \\
& \left.\times \frac{1}{p^{0}-e A^{0}-m} \boldsymbol{\sigma}(\mathbf{p}-e \mathbf{A})\left(p^{0}-e A^{0}-m\right)^{1 / 2}\right] X=0,
\end{aligned}
$$

As before, Eqs. 12 and 13 transform to each other at the replacement of $p^{0} \rightarrow-p^{0}, \mathbf{p} \rightarrow-\mathbf{p}, e \rightarrow-e$ or at the replacement of $+m \rightarrow-m$.

\subsection{C, P, T- symmetries}

Equations 12 and $(13)$ is $P$-invariant at the space reflection $\mathbf{x}^{\prime}=-\mathbf{x}$.

Operations of $\mathrm{C}$ - and T-conjugation are reduced to the operation of the complex conjugation in both cases.

$$
\begin{aligned}
& \Phi^{C}=\Phi^{*}, \quad \Phi^{T}=\Phi^{*}, \\
& \mathrm{X}^{C}=\mathrm{X}^{*}, \quad \mathrm{X}^{T}=\mathrm{X}^{*} .
\end{aligned}
$$

An equation for $\Phi^{C}$ and $\mathrm{X}^{C}$ has the form of

$$
\begin{aligned}
& {\left[\left(p^{0}+e A^{0}\right)^{2}-m^{2}-\left(p^{0}+e A^{0}-m\right)^{1 / 2} \boldsymbol{\sigma}(\mathbf{p}+e \mathbf{A})\right.} \\
& \left.\times \frac{1}{p^{0}+e A^{0}-m} \boldsymbol{\sigma}(\mathbf{p}+e \mathbf{A})\left(p^{0}+e A^{0}-m\right)^{1 / 2}\right] \Phi^{C}=0, \\
& {\left[\left(p^{0}+e A^{0}\right)^{2}-m^{2}-\left(p^{0}+e A^{0}+m\right)^{1 / 2} \boldsymbol{\sigma}(\mathbf{p}+e \mathbf{A})\right.} \\
& \left.\times \frac{1}{p^{0}+e A^{0}+m} \sigma(\mathbf{p}+e \mathbf{A})\left(p^{0}+e A^{0}+m\right)^{1 / 2}\right] \mathrm{X}^{C}=0 .
\end{aligned}
$$

It is easy to see that theory is CPT-invariant. Eq. (14) for $\Phi^{C}$ differs from 12 by the signs before electric charge and mass. If Eq. (12) describes the electrons with charge $-e$ and mass $+m$, then Eq. 14 for $\Phi^{C}$ describes the positrons with charge $+e$ and mass $-m$.

\subsection{Choice of the equations for the quantum electrodynamics}

Each of Eqs. (12)-15) contains solutions with positive and negative energies. In the following, in calculations, we will use only solutions with positive energy. Solutions with negative energy are used only for the mathematical completeness.

We will use solutions of Eqs. (12) and (14) with positive energies for electron and positron. Solutions of Eqs. (13) and (15) correspond to solutions for electrons with mass $-m$ and solutions for positrons with mass $+m$. These solutions do not contain additional physical information compared to the solutions of Eqs. (12) and (14), so, below, we will not consider them. 
Then, in QED, spinor $\Phi(x, s)$ will represent the operator of electron field with positive energy and mass $+m$, spinor $\Phi^{C}(x, s)$ will represent the operator of positron field with positive energy and mass $-m$.

In case of absence of electromagnetic field $\left(A^{\mu}=0\right)$, Eqs. (12) and (14) represent free Klein-Gordon equations with spinor wave functions

$$
\left(p_{0}^{2}-\mathbf{p}^{2}-m^{2}\right) F_{0}^{ \pm}(x, s)=0 .
$$

Here, $F_{0}^{+}(x, s)=\Phi_{0}(x, s)=\Phi_{0}^{C}(x, s)$.

Solutions 16 in the form of plane waves normalized in the continuous spectrum have the form of

$$
F_{0}^{ \pm}(x, p, s)=\frac{1}{\sqrt{(2 \pi)^{3} 2 E_{p}}} e^{\mp i p x} U_{s},
$$

where $E_{p}=p^{0}>0$ and $p^{2}=m^{2}, U_{s}$ are normalized two-component Pauli spin functions.

The conditions of orthonormalization are:

$$
\begin{aligned}
& \int d \mathbf{x} \bar{F}_{0}^{ \pm}\left(x, p^{\prime}, s^{\prime}\right) i \overleftrightarrow{\partial}_{0} F_{0}^{ \pm}(x, p, s)= \pm \delta\left(\mathbf{p}-\mathbf{p}^{\prime}\right) \delta_{s s^{\prime}}, \\
& \int d \mathbf{x} \bar{F}_{0}^{ \pm}\left(x, p^{\prime}, s^{\prime}\right) i \overleftrightarrow{\partial}_{0} F_{0}^{\mp}(x, p, s)=0
\end{aligned}
$$

where denomination of $a \overleftrightarrow{\partial}_{0} b \equiv a \frac{\partial b}{\partial t}-\frac{\partial a}{\partial t} b$. In 18 , the line over the function means Hermitian conjugation.

\section{Formalism of quantum electrodynamics with fermion equations with spinor wave functions}

\subsection{Propagator for Klein-Gordon equation}

The Feynman propagator is found from the solution of the equation of

$$
\left(\square_{x^{\prime}}+m^{2}\right) \Delta_{F}\left(x^{\prime}-x\right)=-\delta^{4}\left(x^{\prime}-x\right),
$$

where $\square_{x^{\prime}}$ is D'Alembertian.

With transition to momentum representation, we obtain (see, for instance, Ref. [9])

$$
\Delta_{F}\left(x^{\prime}-x\right)=\int d^{4} p \frac{e^{-i p\left(x^{\prime}-x\right)}}{(2 \pi)^{4}} \frac{1}{p^{2}-m^{2}+i \varepsilon} .
$$

In our theory, in propagator (20), we use only pole with positive energy.

\section{$3.2 \quad$ Interaction operators}

Let us rewrite Eqs. (12) and (14) as

$$
\begin{aligned}
& \left(\left(p^{0}\right)^{2}-\mathbf{p}^{2}-m^{2}\right) \Phi(x, s)=V\left(p^{\mu},-e A^{\mu},+m\right) \Phi(x, s), \\
& \left(\left(p^{0}\right)^{2}-\mathbf{p}^{2}-m^{2}\right) \Phi^{C}(x, s)=V\left(p^{\mu}, e A^{\mu},-m\right) \Phi^{C}(x, s),
\end{aligned}
$$


where

$$
\begin{aligned}
& V\left(p^{\mu}, \mp e A^{\mu}, \pm m\right)= \pm e\left(p^{0} A^{0}+A^{0} p^{0}\right)-e^{2} A_{0}^{2}-\mathbf{p}^{2} \\
& +\left(p^{0} \mp e A^{0} \pm m\right)^{1 / 2} \boldsymbol{\sigma}(\mathbf{p} \mp e \mathbf{A}) \\
& \times \frac{1}{p^{0} \mp e A^{0} \pm m} \boldsymbol{\sigma}(\mathbf{p} \mp e \mathbf{A})\left(p^{0} \mp e A^{0} \pm m\right)^{1 / 2} .
\end{aligned}
$$

Here, the upper sign corresponds to Eq. (12), the lower sign corresponds to Eq. (14).

As against the Dirac QED representation, our representation leads to the infinite set of interaction vertices depending on the order of the perturbation theory.

In this paper, we will consider QED processes $\sim e, e^{2}$ and $e^{3}$. Let us expand expression 23 to the power of $\sim e^{3}$.

For convenience, let us pass over to the representation where momentum variables are diagonal. In this representation, the matrix element is

$$
\left\langle\mathbf{p}^{\prime}\left|e^{i \mathbf{k x}}\right| \mathbf{p}^{\prime \prime}\right\rangle=\delta\left(\mathbf{p}^{\prime}-\mathbf{p}^{\prime \prime}-\mathbf{k}\right) .
$$

Let us represent the field of $A^{\mu}(\mathbf{x}, t)$ with the Fourier integral. For the case of QED, in denotations of Ref. [11], we have

$$
A^{\mu}(\mathbf{x}, t)=\sum_{\nu= \pm 1} \int A_{(\nu) k}^{\mu}(t) e^{-i \mathbf{k x}} d \mathbf{k}
$$

where

$$
A_{(\nu) k}^{\mu}(t)=\left\{\begin{array}{l}
A_{k}^{\mu} e^{i k_{0} t}, \quad \nu=1, \\
\bar{A}_{(-k)}^{\mu} e^{-i k_{0} t}, \nu=-1 .
\end{array}\right.
$$

In the representation of 24

$$
\begin{aligned}
& \left\langle\mathbf{p}^{\prime}\left|A^{\mu}\right| \mathbf{p}^{\prime \prime}\right\rangle=\sum_{\nu= \pm 1} A_{(\nu)\left(p^{\prime \prime}-p^{\prime}\right)}^{\mu}(t) ;|\mathbf{k}|=\left|\mathbf{p}^{\prime \prime}-\mathbf{p}^{\prime}\right| \\
& \left\langle\mathbf{p}^{\prime}\left|A^{\mu} A^{\lambda}\right| \mathbf{p}^{\prime \prime}\right\rangle=\int d \mathbf{p}^{\prime \prime \prime}\left\langle\mathbf{p}^{\prime}\left|A^{\mu}\right| \mathbf{p}^{\prime \prime \prime}\right\rangle\left\langle\mathbf{p}^{\prime \prime \prime}\left|A^{\lambda}\right| \mathbf{p}^{\prime \prime}\right\rangle \\
& =\sum_{\nu, \nu^{\prime}= \pm 1} d \mathbf{p}^{\prime \prime \prime \prime} A_{(\nu)\left(p^{\prime \prime \prime}-p^{\prime}\right)}^{\mu}(t) A_{\left(\nu^{\prime}\right)\left(p^{\prime \prime}-p^{\prime \prime \prime}\right)}^{\lambda}(t) \text {; } \\
& |\mathbf{k}|=\left|\mathbf{p}^{\prime \prime \prime}-\mathbf{p}^{\prime}\right|,\left|\mathbf{k}^{\prime}\right|=\left|\mathbf{p}^{\prime \prime}-\mathbf{p}^{\prime \prime \prime}\right|, \\
& \left\langle\mathbf{p}^{\prime}\left|A^{\mu} A^{\lambda} A^{\delta}\right| \mathbf{p}^{\prime \prime}\right\rangle=\sum_{\nu, \nu^{\prime}, \nu^{\prime \prime}= \pm 1} \int d \mathbf{p}^{\prime \prime \prime} d \mathbf{p}^{I V} A_{(\nu)\left(p^{\prime \prime \prime}-p^{\prime}\right)}^{\mu}(t) A_{\left(\nu^{\prime}\right)\left(p^{I V}-p^{\prime \prime \prime}\right)}^{\lambda}(t) \\
& \times A_{\left(\nu^{\prime \prime}\right)\left(p^{\prime \prime}-p^{I V}\right)}^{\delta}(t) \text {; } \\
& |\mathbf{k}|=\left|\mathbf{p}^{\prime \prime \prime}-\mathbf{p}^{\prime}\right|, \quad\left|\mathbf{k}^{\prime}\right|=\left|\mathbf{p}^{I V}-\mathbf{p}^{\prime \prime \prime}\right|,\left|\mathbf{k}^{\prime \prime}\right|=\left|\mathbf{p}^{\prime \prime}-\mathbf{p}^{I V}\right| .
\end{aligned}
$$

Let us denote $X=p^{0} \pm m$, where operator $p^{0}=i \frac{\partial}{\partial t}$ as before. Then, in 23,

$$
\begin{aligned}
& \frac{1}{p^{0} \pm m \mp e A^{0}}=\frac{1}{X}+\frac{1}{X}( \pm e) A^{0} \frac{1}{X}+\frac{1}{X}( \pm e) A^{0} \frac{1}{X}( \pm e) A^{0} \frac{1}{X} \\
& +\frac{1}{X}( \pm e) A^{0} \frac{1}{X}( \pm e) A^{0} \frac{1}{X}( \pm e) A^{0} \frac{1}{X}+\ldots
\end{aligned}
$$

At expansion of $\left(p^{0} \mp e A^{0} \pm m\right)^{1 / 2}$ we will use representation 24

$$
\left(X \mp e A^{0}\right)^{1 / 2}=X^{1 / 2}+A+B+C .
$$


Here, the operators are $A \sim e, B \sim e^{2}, C \sim e^{3}$

$$
X \mp e A^{0}=\left(X^{1 / 2}+A+B+C\right)^{2} .
$$

It implies that

1. $A X^{1 / 2}+X^{1 / 2} A=\mp e A^{0}$,

$$
\left\langle\mathbf{p}^{\prime}|A| \mathbf{p}^{\prime \prime}\right\rangle=\left\langle\mathbf{p}^{\prime}\left|\frac{(\mp e) A^{0}}{I+I I}\right| \mathbf{p}^{\prime \prime}\right\rangle,
$$

where $I=\left(X^{\prime}\right)^{1 / 2}, I I=\left(X^{\prime \prime}\right)^{1 / 2}$. In 32 and below we use $\left\langle\mathbf{p}^{\prime}\left|A X^{1 / 2}\right| \mathbf{p}^{\prime \prime}\right\rangle=$ $\left\langle\mathbf{p}^{\prime}|A| \mathbf{p}^{\prime \prime}\right\rangle\left(X^{\prime \prime}\right)^{1 / 2},\left\langle\mathbf{p}^{\prime}\left|X^{1 / 2} A\right| \mathbf{p}^{\prime \prime}\right\rangle=\left(X^{\prime}\right)^{1 / 2}\left\langle\mathbf{p}^{\prime}|A| \mathbf{p}^{\prime \prime}\right\rangle$, etc.

2. $B X^{1 / 2}+X^{1 / 2} B+A^{2}=0$,

$$
\left\langle\mathbf{p}^{\prime}|B| \mathbf{p}^{\prime \prime}\right\rangle=-\int d \mathbf{p}^{\prime \prime \prime} \frac{\left\langle\mathbf{p}^{\prime}\left|( \pm e) A^{0}\right| \mathbf{p}^{\prime \prime \prime}\right\rangle\left\langle\mathbf{p}^{\prime \prime \prime}\left|( \pm e) A^{0}\right| \mathbf{p}^{\prime \prime}\right\rangle}{(I+I I)(I+I I I)(I I I+I I)} .
$$

3. $C X^{1 / 2}+X^{1 / 2} C+B A+A B=0$,

$$
\begin{aligned}
& \left\langle\mathbf{p}^{\prime}|C| \mathbf{p}^{\prime \prime}\right\rangle=-\int d \mathbf{p}^{\prime \prime \prime} d \mathbf{p}^{I V} \\
& \times \frac{(I+I I I+I V+I I)\left\langle\mathbf{p}^{\prime}\left|( \pm e) A^{0}\right| \mathbf{p}^{\prime \prime \prime}\right\rangle\left\langle\mathbf{p}^{\prime \prime \prime}\left|( \pm e) A^{0}\right| \mathbf{p}^{I V}\right\rangle\left\langle\mathbf{p}^{I V}\left|( \pm e) A^{0}\right| \mathbf{p}^{\prime \prime}\right\rangle}{(I+I I)(I+I I I)(I+I V)(I I I+I I)(I V+I I)} .
\end{aligned}
$$

In (33) and 34 III $=\left(X^{\prime \prime \prime}\right)^{1 / 2}, I V=\left(X^{I V}\right)^{1 / 2}$.

Taking into account (30) - (34), sought expansion (23) can be presented as:

$V=V_{1}+V_{2}+V_{3}$, where $V_{1} \sim \pm e, V_{2} \sim e^{2}, V_{3} \sim \pm e^{3}$.

In the momentum representation,

$$
\begin{aligned}
& \left\langle\mathbf{p}^{\prime}\left|V_{1}\right| \mathbf{p}^{\prime \prime}\right\rangle= \pm e\left[\left(\left(p^{\prime}\right)^{0}+\left(p^{\prime \prime}\right)^{0}-\frac{\left(\mathbf{p}^{\prime}\right)^{2}}{I(I+I I)}+\frac{\boldsymbol{\sigma} \mathbf{p}^{\prime} \boldsymbol{\sigma} \mathbf{p}^{\prime \prime}}{I \cdot I I}-\frac{\left(\mathbf{p}^{\prime \prime}\right)^{2}}{I I(I+I I)}\right)\right. \\
& \left.\times\left\langle\mathbf{p}^{\prime}\left|A^{0}\right| \mathbf{p}^{\prime \prime}\right\rangle+\left(-\frac{I I}{I} \boldsymbol{\sigma} \mathbf{p}^{\prime} \sigma^{i}-\frac{I}{I I} \sigma^{i} \boldsymbol{\sigma} \mathbf{p}^{\prime \prime}\right)\left\langle\mathbf{p}^{\prime}\left|A^{i}\right| \mathbf{p}^{\prime \prime}\right\rangle\right] .
\end{aligned}
$$

The expressions for $V_{2}, V_{3}$, having a more cumbersome form, are presented in App. A. For convenience, in (35) and in the formulas of App. A, the factors independent of $A^{\mu}$ are taken outside the matrix element.

\subsection{Feynman rules}

Feynman rules can be determined by means of propagator method [9] and comparison with Feynman rules for scalar electrodynamics of spinless charged particles (see, for example, Ref. [9]). Reference [17] contains the more strict definition of Feynman rules for electrodynamics with spinors in the fermion equations and with Dirac matrixes in spinor representation.

As against the scalar electrodynamics, in our case, there exists the infinite set of interaction vertices with photon depending on the order of the perturbation theory (see $(23))$ : factor $\left(-i V_{1 \mu}\right)$ corresponds to the vertex of interaction with one photon, factor $\left(-i V_{2 \mu \nu}\right)$ corresponds to the vertex of interaction with two photons, etc. For 
convenience, values $V_{1 \mu}, V_{2 \mu \nu}, \ldots$ stand for the appropriate terms of the interaction operator (23) without electromagnetic potentials $A^{\mu}, A^{\mu} A^{\nu}, \ldots$

Each external fermion line corresponds function $F_{0}^{+}(x, p, s)$ (see (17)).

The rest of the Feynman rules are the same as in scalar electrodynamics of charged bosons.

\subsection{Calculations of QED processes}

Taking into account the formulated Feynman rules, we considered some of the QED processes in the first, second and third orders of the perturbation theory. We calculated the matrix elements of Coulomb scattering of electrons, the Møller scattering, Compton effect, and annihilation of electron-positron pair as well as the matrix elements to determine self-energy of an electron, anomalous magnetic moment of electrons, Lamb shift of atomic energy levels.

Figures 1-4 present Feynman diagrams of some of the processes under consideration. Some of the computational details are given in App. B.

The final computational results of the QED amplitudes whose diagrams are given in Figs. 1-3 agree with similar values calculated in Dirac representation.

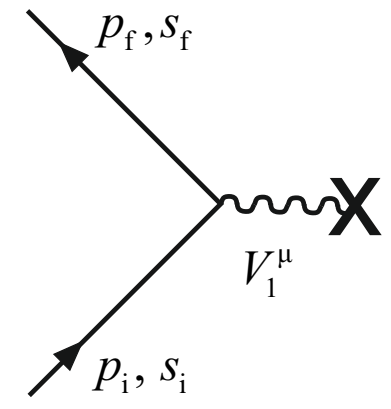

Figure 1: Electron scattering in the Coulomb field

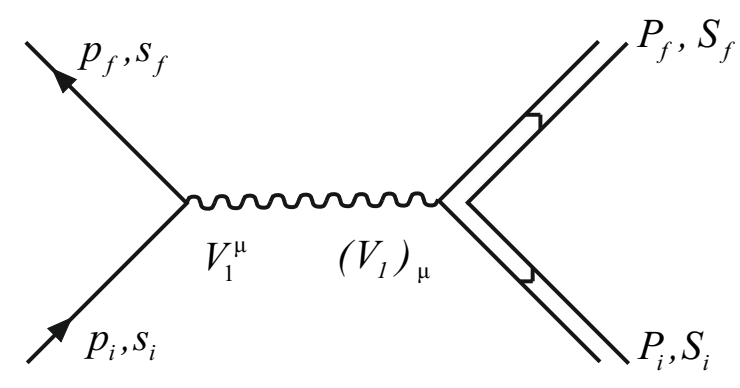

Figure 2: Scattering of an electron on a Dirac proton (Møller scattering)

The calculations of self-energy with ultraviolet logarithmic divergence (see diagrams in Fig.4) also coincide with the results calculated in the Dirac representation. Some of the new nuances are discussed in the following section.

Fig. 5 presents diagrams for radiation corrections to electron scattering in the Coulomb field. Figures 5(a)-5(l) have to be taken into account when calculating the anomalous magnetic moment of an electron (see Sec. 5) and Lamb shift of 


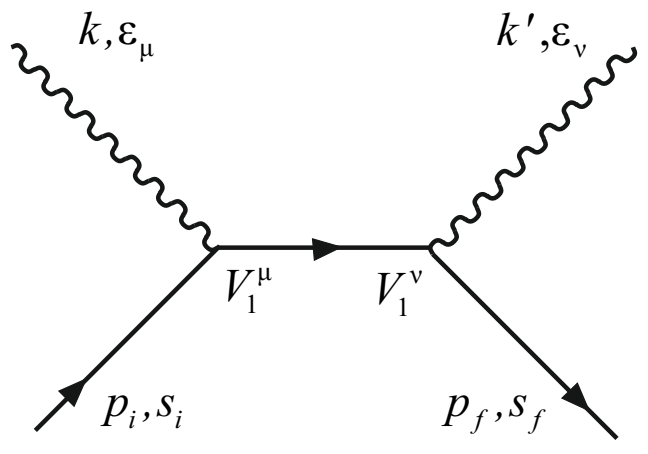

(a)

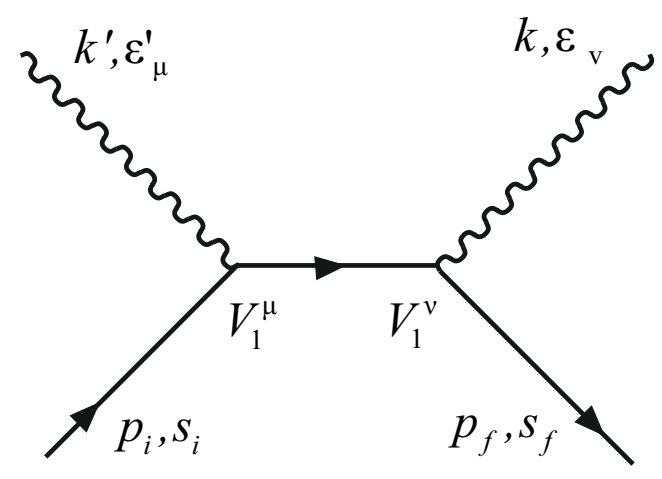

(c)

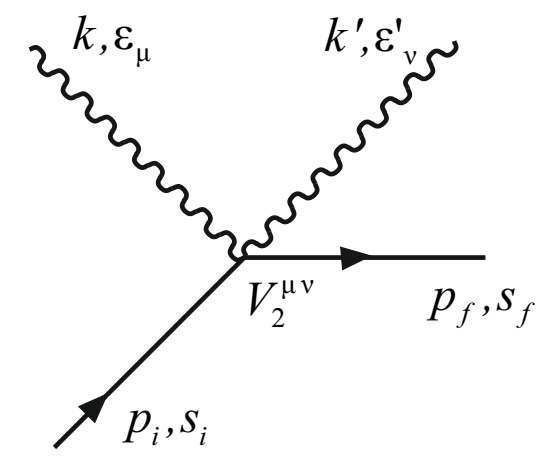

(b)

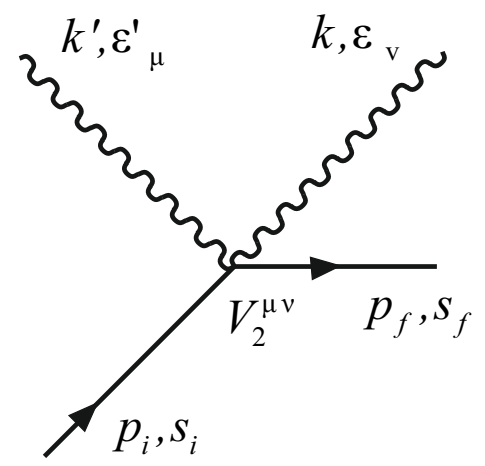

(d)

Figure 3: Compton scattering of electrons

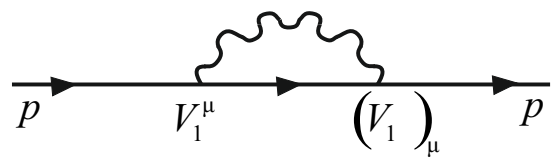

(a)

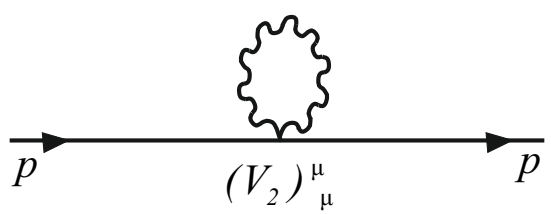

(b)

Figure 4: Self-energy of electron

atomic energy levels (see Sec. 6). In addition, at calculation of the Lamb shift, it is necessary to take into account Figs. $5(\mathrm{~m})-5(\mathrm{o})$ related to the self-energy function of photon用要,

At implementation of renormalization of the electron mass, counterterms corresponding to Figs. 5(a) $-5(\mathrm{~d})$ with $p^{\prime}=p^{I V}$ and Figs. 5(e)-5(h) with $p^{\prime \prime}=p^{\prime \prime \prime}$ have to be subtracted. As a result, the contribution of Figs. 5(c), 5(d), and 5(g), 5(h) is zeroed. The contribution of renormalized Figs. 5(a), 5(b), and 5(e), 5(f) is finite and calculated in Secs. 5, 6. The renormalization of the electron charge is the same as in the standard QED.

\footnotetext{
$\ddagger$ The authors consciously avoid the phrase "vacuum polarization" by reasons stated in Sec. 7 .

§In accordance with structure of interaction operator $V_{3}$ in Fig. $5(1)$, at first, a virtual photon is emitted from vertex, then the interaction with external electromagnetic fields is occurred and further a virtual photon is absorbed in vertex. All photon lines are crossed only in vertex of $\left(V_{3}\right)^{\mu \nu}{ }_{\mu}$
} 


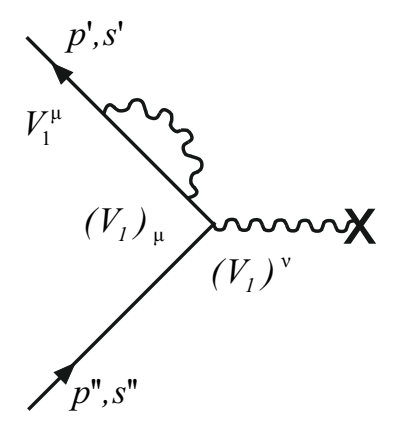

(a)

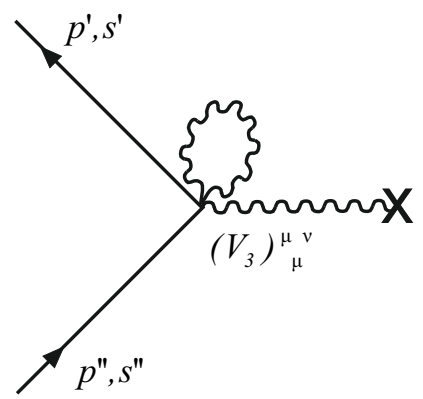

(d)

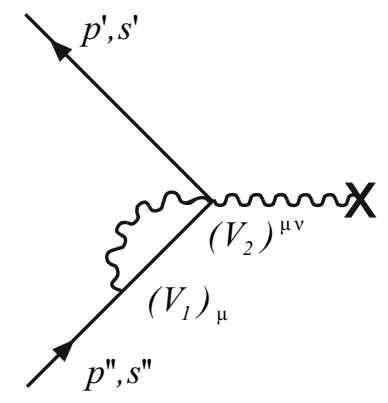

(g)

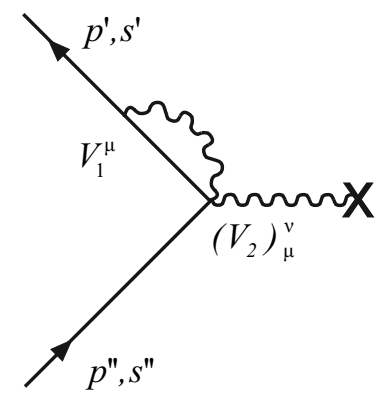

(j)

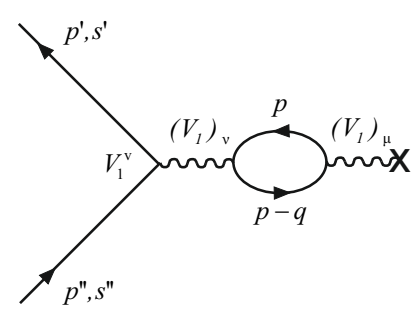

$(\mathrm{m})$

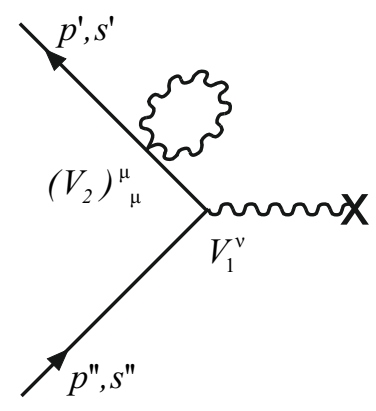

(b)

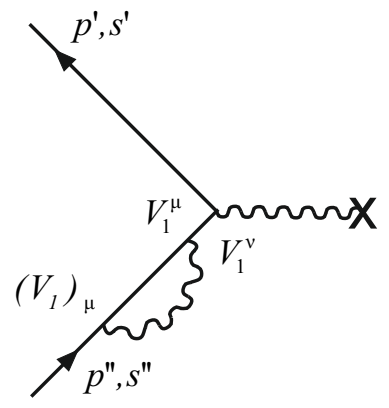

(e)

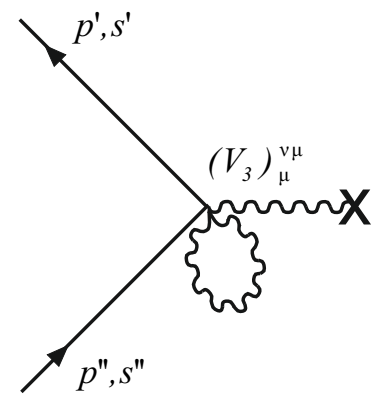

(h)

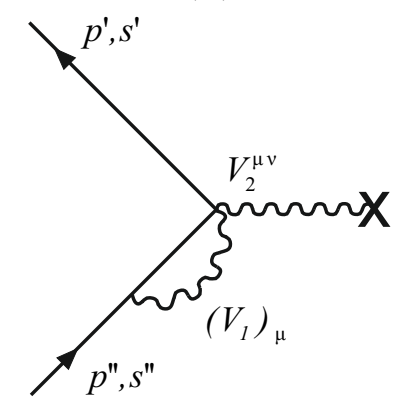

(k)

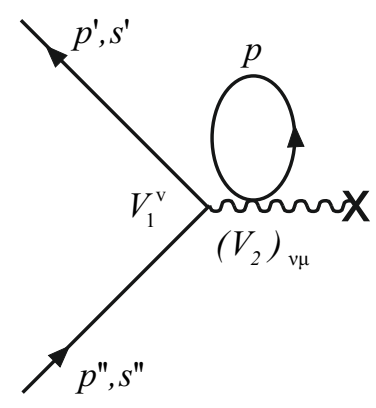

(n)

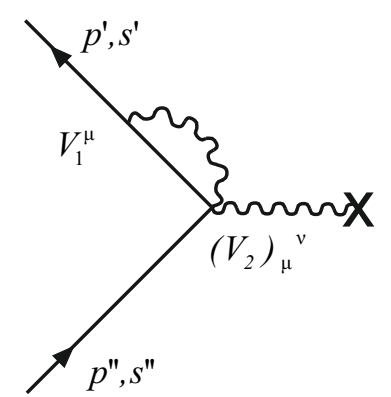

(c)

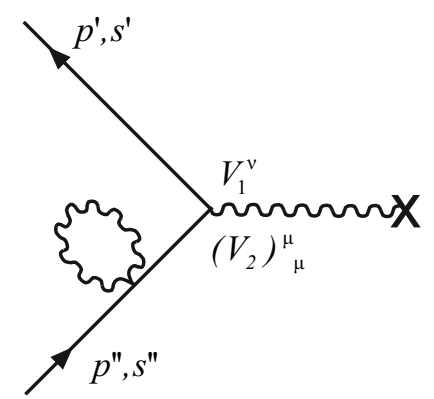

(f)

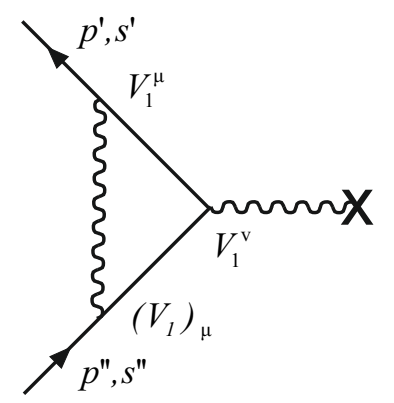

(i)

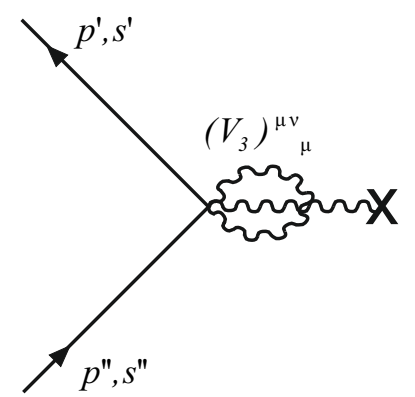

(l)

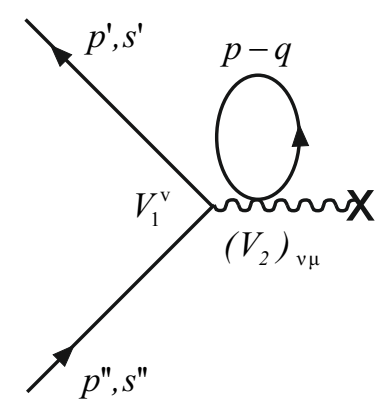

(o)

Figure 5: Radiation corrections to electron scattering in the external field. 


\section{Self-energy of an electron}

The Feynman diagrams to determine self-energy of an electron in the second order of the perturbation theory are presented in Fig.4.

Mass operator $-i \Sigma^{(2)}(p)$ is written as

$$
-i \Sigma^{(2)}(p)=-\int \frac{d^{4} k}{(2 \pi)^{4} k^{2}}\left[V_{1}^{\mu}\left(p^{\prime}, p^{\prime \prime \prime}\right) \frac{1}{\left(p^{\prime \prime \prime}\right)^{2}-m^{2}} V_{1}^{\mu}\left(p^{\prime \prime \prime}, p^{\prime}\right)+V_{2}^{\mu \mu}\left(p^{\prime}, p^{\prime \prime}\right)\right] .
$$

Here, $p^{\prime \prime \prime}=p^{\prime}-k$; for the electron $I=\left(\left(p^{\prime}\right)^{0}+m\right)^{1 / 2}$, $I I I=\left(\left(p^{\prime}\right)^{0}-k^{0}+m\right)^{1 / 2}$. In compliance with 35

$$
\begin{aligned}
& V_{1}^{0}\left(p^{\prime}, p^{\prime \prime \prime}\right)= e\left\{\left(p^{\prime}\right)^{0}+\left(p^{\prime \prime \prime}\right)^{0}-\frac{\left(\mathbf{p}^{\prime}\right)^{2}}{I(I+I I I)}+\frac{\boldsymbol{\sigma} \mathbf{p}^{\prime} \boldsymbol{\sigma} \mathbf{p}^{\prime \prime \prime}}{I \cdot I I I}-\frac{\left(\mathbf{p}^{\prime \prime \prime}\right)^{2}}{I I I(I+I I I)}\right\} \\
&=e\left\{2\left(p^{\prime}\right)^{0}-\right.\left.k^{0}+\frac{2 \mathbf{p}^{\prime} \mathbf{k}}{I I I(I+I I I)}-\frac{k^{2}}{I I I(I+I I I)}-\frac{\boldsymbol{\sigma} \mathbf{p}^{\prime} \boldsymbol{\sigma} \mathbf{k}}{I \cdot I I I}\right\}, \\
& V_{1}^{i}\left(p^{\prime}, p^{\prime \prime \prime}\right)=e\left\{-\frac{I I I}{I} \boldsymbol{\sigma} \mathbf{p}^{\prime} \sigma^{i}-\frac{I}{I I I} \sigma^{i}\left(\boldsymbol{\sigma} \mathbf{p}^{\prime}-\boldsymbol{\sigma} \mathbf{k}\right)\right\} \\
& V_{1}^{0}\left(p^{\prime \prime \prime}, p^{\prime}\right)= e\left\{2\left(p^{\prime}\right)^{0}-k^{0}+\frac{2 \mathbf{p}^{\prime} \mathbf{k}}{I I I(I+I I I)}-\frac{k^{2}}{I I I(I+I I I)}-\frac{\boldsymbol{\sigma} \mathbf{k} \boldsymbol{\sigma} \mathbf{p}^{\prime}}{I \cdot I I I}\right\}, \\
& V_{1}^{i}\left(p^{\prime \prime \prime}, p^{\prime}\right)=e\left\{-\frac{I}{I I I}\left(\boldsymbol{\sigma} \mathbf{p}^{\prime}-\boldsymbol{\sigma} \mathbf{k}\right) \sigma^{i}-\frac{I I I}{I} \sigma^{i} \boldsymbol{\sigma} \mathbf{p}^{\prime}\right\} .
\end{aligned}
$$

In compliance with (A.1)

$$
\begin{gathered}
V_{2}^{00}=e^{2}\left\{-1+\frac{2 \mathbf{p}^{\prime} \mathbf{k}}{I \cdot I I I(I+I I I)^{2}}+\frac{k^{2}}{I I I^{2}(I+I I I)^{2}}\right\} \\
V_{2}^{i k}=\frac{I^{2}}{I I I^{2}} \sigma^{i} \sigma^{k} .
\end{gathered}
$$

Upon substitution in (36) of expressions 37 - 42, we obtain

$$
-i \Sigma^{(2)}(p)=-4 e^{2} \int \frac{d^{4} k}{(2 \pi)^{4} k^{2}} \frac{p k+m^{2}}{(p-k)^{2}-m^{2}} .
$$

The expression (43) agrees with the mass operator in Dirac representation. It is remarkable that expression (43) with ultraviolet logarithmic divergence is obtained from the expression (36) by using only virtual states with positive energy of the electron. In other words, expression (43) is obtained by using only self-conjugated equation (12) with positive energies of an electron. In Dirac representation it would lead to linear divergence of self-energy. In Dirac representation, only accounting of intermediate states with positive and negative energies leads to the view for selfenergy with ultraviolet logarithmic divergence [8].

\section{Anomalous magnetic moment of an electron}

The Feynman diagrams, necessary to calculate corrections to the magnetic moment of an electron in the second order of the perturbation theory, are given in Fig.5. 
In the process under consideration, the static potentials are $A^{i}(\mathbf{x}) \neq 0$, $A^{0}(\mathbf{x})=0$. The amplitude in the first order of the perturbation theory is

$$
-e \frac{i \delta\left(E_{f}-E_{i}\right)}{(2 \pi)^{2} 2 E_{i}} \bar{U}_{s_{f}}\left\langle\mathbf{p}_{f}\left|-\boldsymbol{\sigma} \mathbf{p}_{f} \boldsymbol{\sigma} \mathbf{A}-\boldsymbol{\sigma} \mathbf{A} \boldsymbol{\sigma} \mathbf{p}_{i}\right| \mathbf{p}_{i}\right\rangle>U_{s_{i}} .
$$

Let us denote in 44

$$
L=\boldsymbol{\sigma} \mathbf{p}_{f} \boldsymbol{\sigma} \mathbf{A}+\boldsymbol{\sigma} \mathbf{A} \sigma \mathbf{p}_{i}=\mathbf{p}_{f} \mathbf{A}+\mathbf{A p}_{i}+\boldsymbol{\sigma} \mathbf{H}
$$

It what fallows, we will consider electron motion in the weak magnetic field and take into account only addends proportional to $p^{i}$ and $\boldsymbol{\sigma} \mathbf{H}$, where $\mathbf{H}$ is a magnetic field. In our denotations,

$$
\begin{aligned}
& \mathbf{p}_{f}=\mathbf{p}^{\prime}, \mathbf{p}_{i}=\mathbf{p}^{\prime \prime}, \mathbf{p}^{\prime \prime \prime}=\mathbf{p}^{\prime}-\mathbf{k}, \mathbf{p}^{I V}=\mathbf{p}^{\prime \prime}-\mathbf{k}, \\
& q=p^{\prime}-p^{\prime \prime}=\left\{\begin{array}{l}
q^{0}=0,\left(p^{\prime}\right)^{0}=\left(p^{\prime \prime}\right)^{0} \\
q^{i}=\left(p^{\prime}\right)^{i}-\left(p^{\prime \prime}\right)^{i}
\end{array}\right.
\end{aligned}
$$

Let us consider the contribution of Figs. 5(i)-5(l) to the anomalous magnetic moment. For diagrams of this type, the equation $I I I=I V$ holds true. Let us write down the amplitude as

$$
-\frac{i \delta\left(E^{\prime}-E^{\prime \prime}\right)}{(2 \pi)^{2} 2 E^{\prime \prime}} \bar{U}_{s^{\prime}}\left\langle\mathbf{p}^{\prime}\left|\frac{e^{2}}{(2 \pi)^{4}} \int \frac{d^{4} k}{k^{2}} \Lambda^{i}\left(\mathbf{p}^{\prime}, \mathbf{p}^{\prime \prime}\right) A_{\mathbf{p}^{\prime}-\mathbf{p}^{\prime \prime}}^{i}\right| \mathbf{p}^{\prime \prime}\right\rangle>U_{s^{\prime \prime}}
$$

\subsection{Contribution of Fig. 5(i)}

$$
\begin{aligned}
& \left(\Lambda^{i} A^{i}\right)_{5 i}=\left[V_{1}^{0}\left(p^{\prime}, p^{\prime \prime \prime}\right) V_{1}^{i}\left(p^{\prime \prime \prime}, p^{I V}\right) A^{i} V_{1}^{0}\left(p^{I V}, p^{\prime \prime}\right)\right. \\
& \left.-V_{1}^{l}\left(p^{\prime}, p^{\prime \prime \prime}\right) V_{1}^{i}\left(p^{\prime \prime \prime}, p^{I V}\right) A^{i} V_{1}^{l}\left(p^{I V}, p^{\prime \prime}\right)\right] \frac{1}{\left(\left(p^{\prime \prime \prime}\right)^{2}-m^{2}\right)\left(\left(p^{I V}\right)^{2}-m^{2}\right)} \\
& =e^{3}\left\{-\left(2 m-k^{0}-\frac{\mathbf{k}^{2}}{I I I(I+I I I)}\right)^{2} L\right. \\
& -\left[\left(2 m-k^{0}-\frac{\mathbf{k}^{2}}{I I I(I+I I I)}\right) \frac{1}{I \cdot I I I}+1\right] 2 \mathbf{k} \mathbf{A}\left(\boldsymbol{\sigma} \mathbf{p}^{\prime} \boldsymbol{\sigma} \mathbf{k}+\boldsymbol{\sigma} \mathbf{k} \boldsymbol{\sigma} \mathbf{p}^{\prime \prime}\right) \\
& \left.-\frac{I^{2}}{I I I^{2}} \mathbf{k}^{2} L+\frac{I^{2}}{I I I^{2}}\left[\left(2 \mathbf{p}^{\prime} \mathbf{k}\right) \boldsymbol{\sigma} \mathbf{k} \boldsymbol{\sigma} \mathbf{A}+\left(2 \mathbf{p}^{\prime \prime} \mathbf{k}\right) \boldsymbol{\sigma} \mathbf{A} \boldsymbol{\sigma} \mathbf{k}\right]\right\} \\
& \times \frac{1}{\left[\left(2 m-k^{0}\right) k^{0}+\mathbf{k}^{2}\right]^{2}} .
\end{aligned}
$$




\subsection{Contribution of Figs. $5(\mathrm{j})$ and $5(\mathrm{k})$}

$$
\begin{aligned}
& \left(\Lambda^{i} A^{i}\right)_{5 j}+\left(\Lambda^{i} A^{i}\right)_{5 k}=\left[V_{1}^{0}\left(p^{\prime}, p^{\prime \prime \prime}\right) V_{2}^{i 0}\left(p^{\prime \prime \prime}, p^{\prime \prime}\right) A^{i}\right. \\
& \left.-V_{1}^{l}\left(p^{\prime}, p^{\prime \prime \prime}\right) V_{2}^{i l}\left(p^{\prime \prime \prime}, p^{\prime \prime}\right) A^{i}\right] \frac{1}{\left(p^{\prime \prime \prime}\right)^{2}-m^{2}}+\left[V_{2}^{0 i}\left(p^{\prime}, p^{I V}\right) A^{i} V_{1}^{0}\left(p^{I V}, p^{\prime \prime}\right)\right. \\
& \left.-V_{2}^{l i}\left(p^{\prime}, p^{I V}\right) A^{i} V_{1}^{l}\left(p^{I V}, p^{\prime \prime}\right)\right] \frac{1}{\left(p^{I V}\right)^{2}-m^{2}} \\
& =e^{3}\left\{\left(2 m-k^{0}-\frac{\mathbf{k}^{2}}{I I I(I+I I I)}\right)\left(\frac{2}{I I I(I+I I I)}-\frac{1}{I \cdot I I I}\right) L-L-\frac{I^{2}}{I I I^{2}}\right. \\
& \left.+\frac{1}{I \cdot I I I^{2}(I+I I I)} 2 \mathbf{k} \mathbf{A}\left(\boldsymbol{\sigma} \mathbf{p}^{\prime} \boldsymbol{\sigma} \mathbf{k}+\boldsymbol{\sigma} \mathbf{k} \boldsymbol{\sigma} \mathbf{p}^{\prime \prime}\right)\right\} \frac{1}{\left[-\left(2 m-k^{0}\right) k^{0}-\mathbf{k}^{2}\right]} \\
& -e^{3} \frac{I^{2}}{I I I^{2}}\left[2 \mathbf{p}^{\prime} \mathbf{k} \boldsymbol{\sigma} \mathbf{k} \boldsymbol{\sigma} \mathbf{A}+2 \mathbf{p}^{\prime \prime} \mathbf{k} \boldsymbol{\sigma} \mathbf{A} \boldsymbol{\sigma} \mathbf{k}\right] \frac{1}{\left[\left(2 m-k^{0}\right) k^{0}+\mathbf{k}^{2}\right]^{2}} .
\end{aligned}
$$

\subsection{Contribution of Fig. 5(1)}

$$
\left(\Lambda^{i} A^{i}\right)_{5 l}=V_{3}^{0 i 0} A^{i}=\frac{e^{3} L}{I \cdot I I I(I+I I I)^{2}} .
$$

The sum of expressions 47 - 49

$$
\begin{aligned}
& \left(\Lambda^{i} A^{i}\right)_{5 i+5 j+5 k+5 l} \\
& =-e^{3} \frac{\left(2\left(k^{0}\right)^{2}-2 \mathbf{k}^{2}-8 m k^{0}+4 m^{2}\right) L+4 \mathbf{k} \mathbf{A}\left(\boldsymbol{\sigma} \mathbf{p}^{\prime} \boldsymbol{\sigma} \mathbf{k}+\boldsymbol{\sigma} \mathbf{k} \boldsymbol{\sigma} \mathbf{p}^{\prime \prime}\right)}{\left[\left(2 m-k^{0}\right) k^{0}+\mathbf{k}^{2}\right]^{2}} .
\end{aligned}
$$

The expression (50) coincides with the contribution of single diagram in Fig. 5(i) with vertices $-i e \gamma^{\mu}$ in Dirac representation (see, for example, Ref. [8]).

Let us turn to self-energy diagrams $5(\mathrm{a})-5(\mathrm{~h})$. For each diagrams, we will subtract the appropriate mass counterterm connected with renormalization of electron mass (see Secs. 29, 30 in W.Heitler monograph [8]).

For Figs. 5(a)-5(d), equalities $I=I I=I V$ hold true. For Figs. 5(e)-5(h) equalities $I=I I=I I I$ hold true. If singular denominators do not appear in calculation of contribution from diagrams, then these contributions are completely compensated by appropriate mass counterterms. At appearance of singular denominators, we will use the Heitler limiting process [8]. In these cases, the convergent expressions are remained at subtraction of mass counterterms. In these cases subtractions of mass counterterms lead to finite expressions. 


\subsection{Contribution of Figs. 5(c) and 5(d)}

$$
\begin{aligned}
& \left(\Lambda^{i} A^{i}\right)_{5 c+5 d}=\left[V_{1}^{0}\left(p^{\prime}, p^{\prime \prime \prime}\right) V_{2}^{0 i}\left(p^{\prime \prime \prime}, p^{\prime \prime}\right) A^{i}-V_{1}^{l}\left(p^{\prime}, p^{\prime \prime \prime}\right) V_{2}^{l i}\left(p^{\prime \prime \prime}, p^{\prime \prime}\right) A^{i}\right] \frac{1}{\left(p^{\prime \prime \prime}\right)^{2}-m^{2}} \\
& +V_{3}^{00 i} A^{i}=e^{3}\left[\left(2 m-k^{0}-\frac{\mathbf{k}^{2}}{I I I(I+I I I)}\right)\left(\frac{L}{I(I+I I I)}-\frac{\boldsymbol{\sigma} \mathbf{p}^{\prime} \boldsymbol{\sigma} \mathbf{A}}{I \cdot I I I}\right)-\frac{\mathbf{k}^{2}}{I^{2} I I I^{2}} \boldsymbol{\sigma} \mathbf{p}^{\prime} \boldsymbol{\sigma} \mathbf{A}\right. \\
& +\frac{3 I I I^{2}}{I^{2}} \boldsymbol{\sigma} \mathbf{p}^{\prime} \boldsymbol{\sigma} \mathbf{A}-\boldsymbol{\sigma} \mathbf{p}^{\prime} \boldsymbol{\sigma} \mathbf{A}+\left(2 m-k^{0}-\frac{\mathbf{k}^{2}}{I I I(I+I I I)}\right) \frac{\boldsymbol{\sigma} \mathbf{\sigma} \boldsymbol{A}}{I \cdot I I I}+\boldsymbol{\sigma} \mathbf{k} \boldsymbol{\sigma} \mathbf{A} \\
& \left.+\frac{2 \mathbf{p}^{\prime} \mathbf{k}}{I \cdot I I I^{2}(I+I I I)} \boldsymbol{\sigma} \mathbf{k} \boldsymbol{\sigma} \mathbf{A}\right] \frac{1}{\left(p^{\prime \prime \prime}\right)^{2}-m^{2}}+\frac{e^{3}}{2 I^{2}(I+I I I)^{2}} L \\
& -\frac{e^{3}}{I^{2} \cdot I I I(I+I I I)} \boldsymbol{\sigma} \mathbf{p}^{\prime} \boldsymbol{\sigma} \mathbf{A}-\frac{e^{3}}{I \cdot I I I^{2}(I+I I I)} \boldsymbol{\sigma} \mathbf{k} \mathbf{A} .
\end{aligned}
$$

\subsection{Contribution of Figs. $5(\mathrm{~g})$ and $5(\mathrm{~h})$}

$$
\begin{aligned}
& \left(\Lambda^{i} A^{i}\right)_{5 g+5 h}=\left[V_{2}^{i 0}\left(p^{\prime}, p^{I V}\right) A^{i} V_{1}^{0}\left(p^{I V}, p^{\prime \prime}\right)-V_{2}^{i l}\left(p^{\prime}, p^{I V}\right) A^{i} V_{1}^{l}\left(p^{I V}, p^{\prime \prime}\right)\right] \\
& \times \frac{1}{\left(p^{I V}\right)^{2}-m^{2}}+V_{3}^{i 00} A^{i}=e^{3}\left[\left(2 m-k^{0}-\frac{\mathbf{k}^{2}}{I V(I+I V)}\right)\left(\frac{L}{I(I+I V)}-\frac{\boldsymbol{\sigma} \mathbf{A} \boldsymbol{\sigma} \mathbf{p}^{\prime \prime}}{I \cdot I V}\right)\right. \\
& -\frac{\mathbf{k}^{2}}{I V(I+I V)} \boldsymbol{\sigma} \mathbf{A} \boldsymbol{\sigma} \mathbf{p}^{\prime \prime}+\left(\frac{3 I V^{2}}{I^{2}}-1\right) \boldsymbol{\sigma} \mathbf{A} \boldsymbol{\sigma} \mathbf{p}^{\prime \prime}+\left(2 m-k^{0}-\frac{\mathbf{k}^{2}}{I V(I+I V)}\right) \frac{\boldsymbol{\sigma} \mathbf{A} \boldsymbol{\sigma} \mathbf{k}}{I \cdot I V} \\
& \left.+\boldsymbol{\sigma} \mathbf{A} \boldsymbol{\sigma} \mathbf{k}+\frac{2 \mathbf{p}^{\prime \prime} \mathbf{k}}{I \cdot I V^{2}(I+I V)} \boldsymbol{\sigma} \mathbf{A} \boldsymbol{\sigma} \mathbf{k}\right] \frac{1}{\left(p^{I V}\right)^{2}-m^{2}}+\frac{e^{3}}{2 I^{2}(I+I V)^{2}} L \\
& -\frac{e^{3}}{I^{2} \cdot I V(I+I V)} \boldsymbol{\sigma} \mathbf{A} \boldsymbol{\sigma} \mathbf{p}^{\prime \prime}-e^{3} \frac{\boldsymbol{\sigma} \mathbf{A} \boldsymbol{\sigma} \mathbf{k}}{I \cdot I V^{2}(I+I V)} .
\end{aligned}
$$

Here, $I V=I I I$, since $p_{0}^{I V}=p_{0}^{\prime \prime}-k_{0}=p_{0}^{\prime \prime \prime}=p_{0}^{\prime}-k_{0}$. Taking this into account, the total contribution of (51), (52) is

$$
\begin{aligned}
& \left(\Lambda^{i} A^{i}\right)_{5 c+5 d+5 g+5 h}=e^{3}\left[-\frac{\mathbf{k}^{2} I V(2 I+I I I)}{I^{2} I I I(I+I I I)^{2}}+\frac{3 I I I^{2}}{I^{2}}-1\right] \\
& \times \frac{L}{\left(-\left(2 m-k^{0}\right) k^{0}-\mathbf{k}^{2}\right)}-e^{3} \frac{4\left(\mathbf{p}^{\prime} \mathbf{k} \boldsymbol{\sigma} \mathbf{k} \boldsymbol{\sigma} \mathbf{A}+\mathbf{p}^{\prime \prime} \mathbf{k} \boldsymbol{\sigma} \mathbf{A} \boldsymbol{\sigma} \mathbf{k}\right)}{\left[\left(2 m-k^{0}\right) k^{0}+\mathbf{k}^{2}\right]^{2}} .
\end{aligned}
$$

The equality of $p^{\prime}=p^{I V}$ in Figs. 5(c) and 5(d) and equality of $p^{\prime}=p^{\prime \prime \prime}$ in Figs. 5(g) and $5(\mathrm{~h})$ do not lead to appearance of singular denominators in (51), (52). As a result, contributions of (51), (52) are completely compensated after renormalization of mass by appropriate mass counterterms. 


\subsection{Contribution of Figs. 5(a) and 5(b)}

$$
\begin{aligned}
& \left(\Lambda^{i} A^{i}\right)_{5 a+5 b}=\left\{\left[V_{1}^{0}\left(p^{\prime}, p^{\prime \prime \prime}\right) V_{1}^{0}\left(p^{\prime \prime \prime}, p^{I V}\right) V_{1}^{i}\left(p^{I V}, p^{\prime \prime}\right) A^{i}\right.\right. \\
& \left.-V_{1}^{l}\left(p^{\prime}, p^{\prime \prime \prime}\right) V_{1}^{l}\left(p^{\prime \prime \prime}, p^{I V}\right) A^{i} V_{1}^{i}\left(p^{I V}, p^{\prime \prime}\right)\right] \\
& \times \frac{1}{\left(p^{\prime \prime \prime}\right)^{2}-m^{2}}+V_{2}^{00}\left(p^{\prime}, p^{I V}\right) V_{1}^{i}\left(p^{I V}, p^{\prime \prime}\right) A^{i} \\
& \left.-V_{2}^{l l}\left(p^{\prime}, p^{I V}\right) V_{1}^{i}\left(p^{I V}, p^{\prime \prime}\right) A^{i}\right\} \frac{1}{\left(p^{I V}\right)^{2}-m^{2}} \\
& =e^{3}\left\{\left[\left(p_{0}^{\prime}+p_{0}^{\prime \prime \prime}-\frac{\mathbf{k}^{2}}{I I I(I+I I I)}\right)\right.\right. \\
& \left.\times\left(p_{0}^{\prime \prime \prime}+p_{0}^{I V}-\frac{\mathbf{k}^{2}}{I I I(I I I+I V)}\right)-\frac{3 I \cdot I V}{I I I^{2}} \mathbf{k}^{2}\right] \\
& \left.\times \frac{1}{\left(p^{\prime \prime \prime}\right)^{2}-m^{2}}-1-\frac{3 I \cdot I V}{I I I^{2}}+\frac{\mathbf{k}^{2}}{I I I^{2}(I+I I I)(I I I+I V)}\right\} \\
& \times\left(-\frac{I I}{I V} \boldsymbol{\sigma} \mathbf{p}^{I V} \boldsymbol{\sigma} \mathbf{A}-\frac{I V}{I I} \boldsymbol{\sigma} \mathbf{A} \boldsymbol{\sigma} \mathbf{p}^{\prime \prime}\right) \frac{1}{\left(p^{I V}\right)^{2}-m^{2}} .
\end{aligned}
$$

After algebraic transformations:

$$
\begin{aligned}
& \left(\Lambda^{i} A^{i}\right)_{5 a+5 b}=\left[p_{0}^{\prime} p_{0}^{\prime \prime \prime}+p_{0}^{\prime} p_{0}^{I V}+p_{0}^{\prime \prime \prime}+p_{0}^{I V}\right. \\
& -\frac{\mathbf{k}^{2}}{I I I(I+I I I)}\left(p_{0}^{\prime \prime \prime}+p_{0}^{I V}\right)-\frac{\mathbf{k}^{2}}{I I I(I I I+I V)}\left(p_{0}^{\prime}+p_{0}^{\prime \prime \prime}\right) \\
& \left.+\mathbf{k}^{2}+m^{2}-3 I \cdot I V\left(I I I^{2}-I^{2}\right)+\frac{\mathbf{k}^{2}(I I I-I)}{I I I+I V}\right]\left(-\frac{I I}{I V} \boldsymbol{\sigma} \mathbf{p}^{I V} \boldsymbol{\sigma} \mathbf{A}-\frac{I V}{I I} \boldsymbol{\sigma} \mathbf{A} \boldsymbol{\sigma} \mathbf{p}^{\prime \prime}\right) \\
& \times \frac{1}{\left(p^{\prime \prime \prime}\right)^{2}-m^{2}} \frac{1}{\left(p^{I V}\right)^{2}-m^{2}}=f\left(p_{\mu}^{\prime}-k_{\mu}, p_{\mu}^{\prime}\right) \frac{1}{\left(p^{I V}\right)^{2}-m^{2}} .
\end{aligned}
$$

In (54), (55) $p^{I V}=p^{\prime}$, and we encounter the problem of singular denominator in expression $\frac{\mathcal{P}}{\left(p^{\prime}\right)^{2}-m^{2}}$, where $\mathcal{P}$ is the sign of principal value. To solve the problem, we use the Heitler limiting process [8].

Let us perform substitutions of

$$
\begin{gathered}
p_{\mu}^{\prime \prime \prime} \rightarrow p_{\mu}^{\prime}\left(1+\varepsilon^{\prime}\right)-k_{\mu}, \\
p_{\mu}^{I V} \rightarrow p_{\mu}^{\prime}(1+\varepsilon),
\end{gathered}
$$

where invariants $\varepsilon^{\prime}, \varepsilon$ tend to zero.

Now, let us write down Eq. (55) as

$$
\left(\Lambda^{i} A^{i}\right)_{5 a+5 b}=\int f\left(p_{\mu}^{\prime}\left(1+\varepsilon^{\prime}\right)-k_{\mu}, p_{\mu}^{\prime}(1+\varepsilon)\right) \frac{\mathcal{P}}{m^{2}\left(2 \varepsilon+\varepsilon^{2}\right)} \delta\left(\varepsilon-\varepsilon^{\prime}\right) \delta\left(\varepsilon^{\prime}\right) d \varepsilon d \varepsilon^{\prime} .
$$

To eliminate divergent expressions, let us subtract the mass counterterm

$$
\left(\Lambda^{i} A^{i}\right)_{m}=\int f\left(p_{\mu}^{\prime}-k_{\mu}, p_{\mu}^{\prime}(1+\varepsilon)\right) \frac{\mathcal{P}}{m^{2}\left(2 \varepsilon+\varepsilon^{2}\right)} \delta(\varepsilon) d \varepsilon
$$

In (58) and (59)

$$
\frac{\mathcal{P}}{2 \varepsilon+\varepsilon^{2}}=\frac{1}{2} \frac{\mathcal{P}}{\varepsilon}-\frac{1}{2} \frac{1}{2+\varepsilon},
$$




$$
\frac{1}{2} \frac{\mathcal{P}}{\varepsilon} \delta(\varepsilon)=-\frac{1}{4} \delta^{\prime}(\varepsilon)
$$

In $(55)$ and (59)

$$
\left.f\left(p_{\mu}^{\prime}\left(1+\varepsilon^{\prime}\right)-k_{\mu}, p_{\mu}^{\prime}(1+\varepsilon)\right)\right|_{\varepsilon^{\prime}, \varepsilon=0}=-e^{3} \frac{4 m^{2}+4 m k^{0}}{\left(-\left(2 m-k^{0}\right) k^{0}-\mathbf{k}^{\mathbf{2}}\right)} L .
$$

In what follows, let us denote $M^{2}=4 m^{2}+4 m k^{0}$.

Upon integration in (58) on $\varepsilon^{\prime}$, the total contribution of (58) and (59), taking into account Eqs. (60) and (61), can be written as

$$
\begin{aligned}
& \left(\Lambda^{i} A^{i}\right)_{5 a+5 b+m}=-\frac{1}{4 m^{2}} \int f\left(p_{\mu}^{\prime}(1+\varepsilon), p_{\mu}^{\prime}\right) \times \delta^{\prime}(\varepsilon) d \varepsilon \\
& =\left.\frac{1}{4 m^{2}} \frac{\partial}{\partial \varepsilon} f\left(p_{\mu}^{\prime}(1+\varepsilon), p_{\mu}^{\prime}\right)\right|_{\varepsilon=0}
\end{aligned}
$$

Likewise, we can calculate the contribution of diagrams $5(\mathrm{e})$ and $5(\mathrm{f})$. The total contribution of these diagrams together with the contribution of diagrams $(63)$ is

$$
\left(\Lambda^{i} A^{i}\right)_{S E}=2 e^{3} \frac{2 m^{2}-2 m k^{0}-\left(k^{0}\right)^{2}-\mathbf{k}^{2}}{\left(-2 m k^{0}+\left(k^{0}\right)^{2}-\mathbf{k}^{2}\right)^{2}} L .
$$

The total contribution of all renormalized Figs. 5(a)-5(l) is equal to the sum of expressions (50) and 64

$$
\left(\Lambda^{i} A^{i}\right)_{a m m}=e^{3} \frac{\left(4 m^{2}-4 m k^{0}-2\left(k^{0}\right)^{2}-2 \mathbf{k}^{2}\right) L-4 \mathbf{k} \mathbf{A}\left(\boldsymbol{\sigma} \mathbf{p}^{\prime} \boldsymbol{\sigma} \mathbf{k}+\boldsymbol{\sigma} \mathbf{k} \boldsymbol{\sigma} \mathbf{p}^{\prime \prime}\right)}{\left(-2 m k^{0}+\left(k^{0}\right)^{2}-\mathbf{k}^{2}\right)^{2}} .
$$

The expression (65) coincides with the similar expression in Dirac representation [8. Expression (65) allows to calculate of the anomalous magnetic moment of the electron in the low order of the perturbation theory. The final results agree with the computational results for the anomalous magnetic moment of the electron in the Dirac representation.

\section{$6 \quad$ Lamb shift of energy levels of atomic electrons}

The Feynman diagrams needed to calculate the Lamb shift are presented in Fig. 5.

In the process under consideration, static electromagnetic potentials are $A^{i}(\mathbf{x})=0 . A^{0}(\mathbf{x})=Z e / 4 \pi|\mathbf{x}|$ is the Coulomb potential of the nucleus with atomic number $Z$.

The amplitude of the process in the first order of the perturbation theory is (see in Fig. 1 and (B.1))

$$
S_{f i}=-e \frac{i \delta\left(E_{f}-E_{i}\right)}{(2 \pi)^{2} 2 E_{i}} A^{0}(\mathbf{q}) \bar{U}_{S_{f}}\left\langle\mathbf{p}_{\mathbf{f}}\left|E_{i}+m+\frac{1}{E_{i}+m} \boldsymbol{\sigma} \mathbf{p}_{\mathbf{f}} \boldsymbol{\sigma} \mathbf{p}_{\mathbf{i}}\right| \mathbf{p}_{\mathbf{i}}\right\rangle U_{S_{i}} .
$$

Here, $A^{0}(\mathbf{q})=4 \pi /\left(\mathbf{q}^{2}\right)$.

In our denotations,

$$
\begin{aligned}
& \mathbf{p}_{\mathbf{f}}=\mathbf{p}^{\prime}, \mathbf{p}_{\mathbf{i}}=\mathbf{p}^{\prime \prime}, \mathbf{p}^{\prime \prime \prime}=\mathbf{p}^{\prime}-\mathbf{k}, \mathbf{p}^{\mathbf{I V}}=\mathbf{p}^{\prime \prime}-\mathbf{k}, \\
& q=p^{\prime}-p^{\prime \prime}=\left\{\begin{array}{l}
q^{0}=0,\left(p^{\prime}\right)^{0}=\left(p^{\prime \prime}\right)^{0} \\
q^{i}=\left(p^{\prime}\right)^{i}-\left(p^{\prime \prime}\right)^{i}
\end{array}\right.
\end{aligned}
$$


In the following, we will consider electron motion in the Coulomb field in the non-relativistic approximation and take into account the addends up to $\left(\mathbf{p}^{\prime}\right)^{2},\left(\mathbf{p}^{\prime \prime}\right)^{2}$.

Let us consider the contribution of Figs. 5(i) $-5(\mathrm{l})$ to the Lamb shift (see Fig. 5). For this type of diagrams the equality of $I I I=I V$ holds true. We write down the amplitude as

$$
-e \frac{i \delta\left(E^{\prime}-E^{\prime \prime}\right)}{(2 \pi)^{2} 2 E^{\prime \prime}} \bar{U}_{S^{\prime}}\left\langle\mathbf{p}^{\prime}\left|\frac{1}{(2 \pi)^{4}} \int \frac{d^{4} k}{k^{2}} \Lambda^{0}\left(\mathbf{p}^{\prime}, \mathbf{p}^{\prime \prime}\right) A^{0}(\mathbf{q})\right| \mathbf{p}^{\prime \prime}\right\rangle U_{S^{\prime \prime}}
$$

\subsection{Contribution of Fig. 5(i)}

$$
\begin{aligned}
& \left(\Lambda^{0} A^{0}\right)_{5 i}=\left[V_{1}^{0}\left(p^{\prime}, p^{\prime \prime \prime}\right) V_{1}^{0}\left(p^{\prime \prime \prime}, p^{I V}\right) A^{0} V_{1}^{0}\left(p^{I V}, p^{\prime \prime}\right)\right. \\
& \left.-V_{1}^{i}\left(p^{\prime}, p^{\prime \prime \prime}\right) V_{1}^{0}\left(p^{\prime \prime \prime}, p^{I V}\right) A^{0} V_{1}^{i}\left(p^{I V}, p^{\prime \prime}\right)\right] \frac{1}{\left(\left(p^{\prime \prime \prime}\right)^{2}-m^{2}\right)\left(\left(p^{I V}\right)^{2}-m^{2}\right)} .
\end{aligned}
$$

The expression (68) with substitution of $V_{1}^{0}, V_{1}^{i}$ has a cumbersome form and is given in the App. C (see (C.1)).

\subsection{Contribution of Figs. 5(j) and 5(k)}

$$
\begin{aligned}
& \left(\Lambda^{0} A^{0}\right)_{5 j}+\left(\Lambda^{0} A^{0}\right)_{5 k}=\left[V_{1}^{0}\left(p^{\prime}, p^{\prime \prime \prime}\right) V_{2}^{00}\left(p^{\prime \prime \prime}, p^{\prime \prime}\right) A^{0}\right. \\
& \left.-V_{1}^{i}\left(p^{\prime}, p^{\prime \prime \prime}\right) V_{2}^{0 i}\left(p^{\prime \prime}, p^{\prime \prime}\right) A^{0}\right] \frac{1}{\left(p^{\prime \prime \prime}\right)^{2}-m^{2}}+\left[V_{2}^{00}\left(p^{\prime}, p^{I V}\right) A^{0} V_{1}^{0}\left(p^{I V}, p^{\prime \prime}\right)\right. \\
& \left.-V_{2}^{i 0}\left(p^{\prime}, p^{I V}\right) A^{0} V_{1}^{i}\left(p^{I V}, p^{\prime \prime}\right)\right] \frac{1}{\left(p^{I V}\right)^{2}-m^{2}} .
\end{aligned}
$$

\subsection{Contribution of Fig. 5(1)}

$$
\left(\Lambda^{0} A^{0}\right)_{5 l}=V_{3}^{000} A^{0}-V_{3}^{i 0 i} A^{0}
$$

The final expressions (69), (70) are given in the App. C (see Eqs. (C.2), (C.3)).

The sum of (C.1)-(C.3) accurate to $\left(\mathbf{p}^{\prime}\right)^{2},\left(\mathbf{p}^{\prime \prime}\right)^{2}$ was determined by using software package "Maple". As a result, the total contribution of Figs. 5(i)-5(l) can be written as

$$
\begin{aligned}
& \left(\Lambda^{0} A^{0}\right)_{5 i+5 j+5 k+5 l}=2 e^{3} m\left[4 m^{2}-2\left(k^{0}\right)^{2}-2 \mathbf{k}^{2}-4 m k^{0}\right. \\
& -\frac{k^{2}+\left(k^{0}\right)^{2}+2 m k^{0}-10 m^{2}}{4 m^{2}}\left(\left(\mathbf{p}^{\prime}\right)^{2}+\left(\mathbf{p}^{\prime \prime}\right)^{2}\right)-4 \mathbf{p}^{\prime} \mathbf{p}^{\prime \prime}+4\left(\mathbf{p}^{\prime} \mathbf{k}+\mathbf{p}^{\prime \prime} \mathbf{k}\right) \\
& +\left(\frac{2 k^{0}}{m}-4\right)\left(\boldsymbol{\sigma} \mathbf{p}^{\prime} \boldsymbol{\sigma} \mathbf{k}+\boldsymbol{\sigma} \mathbf{k} \boldsymbol{\sigma} \mathbf{p}^{\prime \prime}\right) \\
& \left.+\frac{1}{4 m^{2}}\left(4 m^{2}-2\left(k^{0}\right)^{2}-2 \mathbf{k}^{2}-4 m k^{0}\right) \boldsymbol{\sigma} \mathbf{p}^{\prime} \boldsymbol{\sigma} \mathbf{p}^{\prime \prime}\right] A^{0}(\mathbf{q}) .
\end{aligned}
$$

In the standard QED, the similar contribution to the Lamb shift is provided by the only 5(i) with vertexes $-i e \gamma^{\mu}$. The analog of (71) is (see, for example, Ref. [12])

$$
\begin{aligned}
& \left(\Lambda^{0} A^{0}\right)_{D i r}=e^{3}\left\{\left(4 m^{2}+2 \mathbf{q}^{2}-2 \mathbf{k}^{2}-2\left(k^{0}\right)^{2}\right) \gamma^{0}\right. \\
& \left.+4 i \boldsymbol{\sigma}(\mathbf{k} \times \mathbf{q}) \gamma^{0}+k^{0}(-4 m+4 \boldsymbol{\gamma} \mathbf{k})\right\} A^{0}(\mathbf{q}) .
\end{aligned}
$$


In the scattering amplitude, expression $(72)$ will be found sandwiched between bispinors $\Psi\left(p^{\prime \prime}\right)$ and $\bar{\Psi}\left(p^{\prime}\right) \gamma^{0}$, where

$$
\Psi\left(p^{\prime \prime}\right)=\frac{1}{\sqrt{2\left(p^{\prime \prime}\right)^{0}}} \sqrt{\left(p^{\prime \prime}\right)^{0}+m}\left(\begin{array}{c}
\varphi\left(p^{\prime \prime}\right) \\
\frac{\boldsymbol{\sigma} \mathbf{p}^{\prime \prime}}{\left(p^{\prime \prime}\right)^{0}+m} \varphi\left(p^{\prime \prime}\right)
\end{array}\right) .
$$

Expanding 73 and $\bar{\Psi}\left(p^{\prime}\right) \gamma^{0}$ in powers not higher than $\left(\mathbf{p}^{\prime}\right)^{2},\left(\mathbf{p}^{\prime \prime}\right)^{2}$ and considering, with our accuracy, that spinors are $\varphi\left(p^{\prime}\right)=2 m \Phi\left(p^{\prime}\right), \varphi\left(p^{\prime \prime}\right)=2 m \Phi\left(p^{\prime \prime}\right)$ (see (10) and (11)), we obtain that the expression (72) sandwiched between spinors $\Phi\left(p^{\prime}\right), \Phi\left(p^{\prime \prime}\right)$ coincides with 71 .

Let us note that expression $\frac{1}{\sqrt{\left(p^{\prime \prime}\right)^{0}}}$ in 73 , as well as expression $\frac{1}{\sqrt{\left(p^{\prime}\right)^{0}}}$ in

Hermitian-conjugated spinor are not subjected to expansion since in amplitude (67) both the expressions are present in multiplied form of $\frac{1}{2 E^{\prime \prime}}$.

\subsection{Contribution of Figs. $5(\mathrm{a})-5(\mathrm{~h})$}

Now, let us consider to the contribution of self-energy diagrams $5(\mathrm{a})-5(\mathrm{~h})$. For Figs. $5(\mathrm{a})-5(\mathrm{~d})$ equalities $I=I I=I V$ hold true. For Figs. $5(\mathrm{e})-5(\mathrm{~h})$, equalities $I=I I=I I I$ hold true. The equality $p^{\prime}=p^{I V}$ in diagrams 5(c) and 5(d) and equality $p^{\prime \prime}=p^{\prime \prime \prime}$ in Figs. $5(\mathrm{~g})$ and $5(\mathrm{~h})$ do not lead to appearance of singular denominators in calculations. As a result, the contributions of Figs. 5(c), 5(d), and $5(\mathrm{~g}), 5(\mathrm{~h})$ are completely compensated for after renormalization of mass by appropriate mass counterterms. The contribution of Figs. 5(a), 5(b), and 5(e), 5(f) is determined by the expression

$$
\begin{aligned}
& \left(\Lambda^{0} A^{0}\right)_{5 a+5 b+5 e+5 f}=\left\{\left[V_{1}^{0}\left(p^{\prime}, p^{\prime \prime \prime}\right) V_{1}^{0}\left(p^{\prime \prime \prime}, p^{I V}\right)-V_{1}^{i}\left(p^{\prime}, p^{\prime \prime \prime}\right) V_{1}^{i}\left(p^{\prime \prime \prime}, p^{I V}\right)\right]\right. \\
& \left.\times \frac{1}{\left(p^{\prime \prime \prime}\right)^{2}-m^{2}}+V_{2}^{00}\left(p^{\prime}, p^{I V}\right)-V_{2}^{l l}\left(p^{\prime}, p^{I V}\right)\right\} \frac{V_{1}^{0}\left(p^{I V}, p^{\prime \prime}\right) A^{0}}{\left(p^{I V}\right)^{2}-m^{2}} \\
& +\left\{\left[V_{1}^{0}\left(p^{\prime \prime \prime}, p^{I V}\right) V_{1}^{0}\left(p^{I V}, p^{\prime \prime}\right)-V_{1}^{i}\left(p^{\prime \prime \prime}, p^{I V}\right) V_{1}^{i}\left(p^{I V}, p^{\prime \prime}\right)\right]\right. \\
& \left.\times \frac{1}{\left(p^{I V}\right)^{2}-m^{2}}+V_{2}^{00}\left(p^{\prime \prime \prime}, p^{\prime \prime}\right)-V_{2}^{l l}\left(p^{\prime \prime \prime}, p^{\prime \prime}\right)\right\} \frac{V_{1}^{0}\left(p^{\prime}, p^{\prime \prime \prime}\right) A^{0}}{\left(p^{\prime \prime \prime}\right)^{2}-m^{2}}
\end{aligned}
$$

For Figs. 5(a), 5(b), and 5(e), 5(f), there is a problem of singular denominators. In expression (74), these denominators stand after curly brackets. To solve the problem, we used the Heitler limiting process [8] as in Sec. 5. Performing calculations similar to the calculations in Sec. 5 (see (54)-(64)), we will obtain the total contribution of self-energy diagrams

$$
\left(\Lambda^{0} A^{0}\right)_{S E}=2 e^{3} \frac{2 m^{2}+\mathbf{k}^{2}-2\left(p^{\prime}\right)^{0} k^{0}+2 \mathbf{p}^{\prime} \mathbf{k}-\frac{2}{m^{2}}\left(\left(p^{\prime}\right)^{0} k^{0}-\mathbf{p}^{\prime} \mathbf{k}\right)^{2}}{\left[\left(\left(p^{\prime}\right)^{0}-k^{0}\right)^{2}-\left(\mathbf{p}^{\prime}-\mathbf{k}\right)^{2}-m^{2}\right]^{2}} A^{0}(\mathbf{q}) .
$$

The expression (75) coincides with the similar expression in Dirac representation (see for example, Ref. [8]). The total contribution of Figs. 5(a)-5(h) to the Lamb shift of atomic levels coincides with the similar contribution of diagrams in the Dirac representation with vertexes $-i e \gamma^{\mu}$. 


\subsection{Contribution of Figs. 5(m)-5(o)}

Diagrams $5(\mathrm{~m})-5(\mathrm{o})$ are connected with self-energy function of photon (see, for example, Ref. [16]). In the second order of the perturbation theory, the photon propagator is written in the form of

$$
-i D_{\alpha \beta}=\frac{(-i) g_{\alpha \beta}}{q^{2}}+\frac{(-i) g_{\alpha \mu}}{q^{2}} i \Pi_{\mu \nu} \frac{(-i) g_{\nu \beta}}{q^{2}} .
$$

In compliance with diagrams $5(\mathrm{~m})-5(\mathrm{o})$, the tensor $\Pi_{\mu \nu}$ has the form of

$$
\begin{aligned}
& \Pi_{\mu \nu}(q)=i 4 \pi e^{2} \int \frac{d^{4} p}{(2 \pi)^{4}} \operatorname{Tr}\left[\frac{V_{1 \mu}(p, p-q) V_{1 \nu}(p-q, p)+V_{1 \mu}(p-q, p) V_{1 \nu}(p, p-q)}{2\left(p^{2}-m^{2}\right)\left((p-q)^{2}-m^{2}\right)}\right. \\
& \left.+\frac{V_{2 \mu \nu}(p-q, p, p-q)}{(p-q)^{2}-m^{2}}+\frac{V_{2 \mu \nu}(p, p-q, p)}{p^{2}-m^{2}}\right] .
\end{aligned}
$$

At electron scattering in the static external field $A_{0}(\mathbf{x})$ only the component $\Pi_{00}(q)$ contributes to amplitude of process. In this case $q_{0}=0$. Then

$$
\begin{gathered}
f\left(p^{0}\right)=\operatorname{Tr}\left[V_{10}(p, p-q) V_{10}(p-q, p)\right]=S p\left[V_{10}(p-q, p) V_{10}(p, p-q)\right] \\
=2 e^{2}\left[\left(2 p^{0}-\frac{\mathbf{p}^{2}}{2\left(p^{0}+m\right)}-\frac{\mathbf{p}_{1}^{2}}{2\left(p^{0}+m\right)}\right)^{2}\right. \\
\left.\quad+\frac{2 \mathbf{p p}_{1}}{p^{0}+m}\left(2 p^{0}-\frac{\mathbf{p}_{1}^{2}}{2\left(p^{0}+m\right)}-\frac{\mathbf{p}^{2}}{2\left(p^{0}+m\right)}\right)+\frac{\mathbf{p}^{2} \mathbf{p}_{1}^{2}}{\left(p^{0}+m\right)^{2}}\right], \\
\operatorname{Tr} V_{2}^{00}(p, p-q, p)=2 e^{2}\left[-1+\frac{3}{4} \frac{\mathbf{p}^{2}}{\left(p^{0}+m\right)^{2}}+\frac{1}{4} \frac{\mathbf{p}_{1}^{2}}{\left(p^{0}+m\right)^{2}}-\frac{\mathbf{p p}_{1}}{\left(p^{0}+m\right)^{2}}\right] \\
\operatorname{Tr}_{2}^{00}(p-q, p, p-q)=2 e^{2}\left[-1+\frac{1}{4} \frac{\mathbf{p}^{2}}{\left(p^{0}+m\right)^{2}}+\frac{3}{4} \frac{\mathbf{p}_{1}^{2}}{\left(p^{0}+m\right)^{2}}-\frac{\mathbf{p p}_{1}}{\left(p^{0}+m\right)^{2}}\right] .
\end{gathered}
$$

In 78$)-(80)$

$$
\begin{aligned}
& \Pi_{00}(q)=-\frac{4 \pi e^{2}}{(2 \pi)^{3}} \int d \mathbf{p} \operatorname{Tr}\left\{\frac{f\left(p^{0}=E_{1}\right)}{2 E_{1}\left(E_{1}^{2}-E^{2}\right)}-\frac{f\left(p^{0}=E\right)}{2 E\left(E_{1}^{2}-E^{2}\right)}\right. \\
& \left.+\left.V_{2}^{00}(p-q, p, p-q)\right|_{p^{0}=E_{1}} \frac{1}{2 E_{1}}+\left.V_{2}^{00}(p, p-q, p)\right|_{p^{0}=E} \frac{1}{2 E}\right\} .
\end{aligned}
$$

The expression (81) is obtained by contour integration of (77) with respect to variable $\mathrm{d} p^{0}$ with bypass of poles with positive energy $p^{0}=E=\left(m^{2}+\mathbf{p}^{2}\right)^{1 / 2}$ and $p^{0}=E_{1}=\left(m^{2}+(\mathbf{p}-\mathbf{q})^{2}\right)^{1 / 2}$.

After algebraic transformations, the expression 81 can be written in the form of

$$
\Pi_{00}(q)=\frac{e^{2}}{\pi^{2}} \int d \mathbf{p} \frac{-E E_{1}+\mathbf{p p}_{1}+m^{2}}{E E_{1}\left(E+E_{1}\right)} .
$$

The expression 82 coincides with the tensor $\Pi_{00}(q)$ in standard QED (see, for example, Ref. [8]).

Thus, the total contribution of diagrams 5(a)-5(o) in the Lamb shift of atomic levels coincides with the appropriate contribution of diagrams in the standard QED with vertices $-i e \gamma^{\mu}$.

In the developed theory, it is not necessary to include creation and annihilation of virtual electron-positron pairs in the calculations while defining physical effects. Let us adduce some arguments. 
1. The final calculation results of Figs. 5(m)-5(o) connected with self-energy function of a photon are obtained by using only states with positive energies of electrons in intermediate states.

2. Diagram $5(\mathrm{~m})$ can be considered as vacuum polarization by virtual electronpositron pairs but the Figs. 5(n) and 5(o) cannot be interpreted in such manner. However these two diagrams are required to determine, for example, Lamb shift at using Klein-Gordon equation with spinor functions for fermions [9, 17].

\section{Conclusions}

In the paper, quantum electrodynamics is considered with self-conjugated equations with spinor wave functions for fermion fields.

For the considered QED, some of Feynman diagrams are calculated. In the low order of the perturbation theory, the matrix elements of Coulomb scattering of electrons, scattering of an electron on a proton (Møller scattering), Compton effect, and annihilation of the electron-positron pair are calculated. The final results coincide with the similar values calculated in the standard QED when using the Dirac equation with the bispinor wave functions.

Self-energy of an electron with ultraviolet logarithmic divergence coincides with the value computed in the standard QED. New feature is the absence of contribution to the self-energy of intermediate states with negative energies. In the standard QED in this case, the self-energy linearly diverges in the ultraviolet limit. Only accounting of the contribution of the intermediate states with positive and negative energies leads to the ultraviolet logarithmic divergence of self-energy [8]. The only intermediate states with positive energies are also used while calculating the matrix elements related to the anomalous magnetic moment and the Lamb shift of electron.

The calculated values of the matrix elements for determining the anomalous magnetic moment of the electron and Lamb shift are consistent with the matrix elements determined in the standard QED.

In the QED under consideration, there are no operators connecting solutions with positive and negative energies for fermions. The equations for electrons and positrons are not connected with each other.

In the developed theory, while defining the physical effects, we do not need to introduce the concept of vacuum polarization and calculate creation and annihilation of virtual electron-positron pairs. In QED under consideration, in the equations, the masses of particles and antiparticles have the opposite signs. For the first time one of the authors showed this in 1989 (see Ref. [13]-[15]). Later, other researchers came to the same conclusion (see, for example, Refs. [18]-[20]). In a certain sense, emergence of negative mass in our theory is the result of refusal to use states with negative energies (see Eqs. (12), (14) or (13), (15)). The future will show possibility of proving a more profound physical significance of this fact.

\section{Acknowledgments}

The authors express their gratitude to A.L.Novoselova for essential technical assistance in preparation of the paper. 
APPENDIX A. Interaction operators $\sim e^{2}, e^{3}$

$$
\begin{aligned}
& V_{2}=e^{2} \int d \mathbf{p}^{\prime \prime \prime}\left\{\left[-1-\frac{\left(\mathbf{p}^{\prime}\right)^{2}}{I(I+I I)(I+I I I)(I I I+I I)}\right.\right. \\
& +\frac{\left(\mathbf{p}^{\prime \prime \prime}\right)^{2}}{I I I^{2}(I+I I I)(I I+I I I)}-\frac{\left(\mathbf{p}^{\prime \prime}\right)^{2}}{I I(I+I I)(I+I I I)(I I I+I I)} \\
& \left.+\frac{\boldsymbol{\sigma} \mathbf{p}^{\prime} \boldsymbol{\sigma} \mathbf{p}^{\prime \prime}}{I \cdot I I \cdot I I I^{2}}-\frac{\boldsymbol{\sigma} \mathbf{p}^{\prime} \boldsymbol{\sigma} \mathbf{p}^{\prime \prime \prime}}{I I \cdot I I I^{2} \cdot(I I+I I I)}-\frac{\boldsymbol{\sigma} \mathbf{p}^{\prime \prime \prime} \boldsymbol{\sigma} \mathbf{p}^{\prime \prime}}{I I \cdot I I I^{2} \cdot(I+I I I)}\right] \\
& \times\left\langle\mathbf{p}^{\prime}\left|A^{0}\right| \mathbf{p}^{\prime \prime \prime}\right\rangle\left\langle\mathbf{p}^{\prime \prime \prime}\left|A^{0}\right| \mathbf{p}^{\prime \prime}\right\rangle+\frac{I \cdot I I}{I I I^{2}} \sigma^{i} \sigma^{k}\left\langle\mathbf{p}^{\prime}\left|A^{i}\right| \mathbf{p}^{\prime \prime \prime}\right\rangle\left\langle\mathbf{p}^{\prime \prime \prime}\left|A^{k}\right| \mathbf{p}^{\prime \prime}\right\rangle \\
& +\left[\frac{\boldsymbol{\sigma} \mathbf{p}^{\prime} \sigma^{i}}{I(I+I I I)}-\frac{I \sigma^{i} \boldsymbol{\sigma} \mathbf{p}^{\prime \prime}}{I I \cdot I I I^{2}}+\frac{I \sigma^{i} \boldsymbol{\sigma} \mathbf{p}^{\prime \prime \prime}}{I I I^{2}(I I I+I I)}\right]\left\langle\mathbf{p}^{\prime}\left|A^{i}\right| \mathbf{p}^{\prime \prime \prime}\right\rangle\left\langle\mathbf{p}^{\prime \prime \prime}\left|A^{0}\right| \mathbf{p}^{\prime \prime}\right\rangle \\
& +\left[-\frac{I I}{I \cdot I I I^{2}} \boldsymbol{\sigma} \mathbf{p}^{\prime} \sigma^{i}+\frac{1}{I I(I+I I I)} \sigma^{i} \boldsymbol{\sigma} \mathbf{p}^{\prime \prime}+\frac{I I}{I I I^{2}(I+I I I)} \boldsymbol{\sigma} \mathbf{p}^{\prime \prime \prime} \sigma^{i}\right] \\
& \left.\times\left\langle\mathbf{p}^{\prime}\left|A^{0}\right| \mathbf{p}^{\prime \prime \prime}\right\rangle\left\langle\mathbf{p}^{\prime \prime \prime}\left|A^{i}\right| \mathbf{p}^{\prime \prime}\right\rangle\right\}, \\
& V_{3}= \pm e^{3} \int d \mathbf{p}^{\prime \prime \prime} d \mathbf{p}^{I V}\left\{\left(\frac{\left(\mathbf{p}^{I V}\right)^{2}}{I V^{2} \cdot(I+I V)(I+I I I)(I I I+I V)(I V+I I)}\right.\right. \\
& -\frac{\boldsymbol{\sigma} \mathbf{p}^{\prime} \boldsymbol{\sigma} \mathbf{p}^{I V}}{I \cdot I I I^{2} \cdot I V^{2}(I V+I I)}-\frac{\boldsymbol{\sigma} \mathbf{p}^{\prime \prime \prime} \boldsymbol{\sigma} \mathbf{p}^{\prime \prime}}{I I \cdot I I I^{2} \cdot I V^{2}(I+I I I)} \\
& +\frac{\left(\mathbf{p}^{\prime \prime \prime}\right)^{2}}{I I I^{2} \cdot(I+I I I)(I I I+I V)(I I I+I I)(I V+I I)} \\
& -\frac{\boldsymbol{\sigma} \mathbf{p}^{I V} \boldsymbol{\sigma} \mathbf{p}^{\prime \prime}}{I I \cdot I V^{2}(I+I I I)(I+I V)(I I I+I V)} \\
& -\frac{\boldsymbol{\sigma} \mathbf{p}^{\prime} \boldsymbol{\sigma} \mathbf{p}^{\prime \prime \prime}}{I \cdot I I I^{2}(I I I+I I)(I I I+I V)(I V+I I)} \\
& +\frac{\boldsymbol{\sigma} \mathbf{p}^{\prime \prime \prime} \boldsymbol{\sigma} \mathbf{p}^{I V}}{I I I^{2} \cdot I V^{2}(I+I I I)(I V+I I)}+\frac{\boldsymbol{\sigma} \mathbf{p}^{\prime} \boldsymbol{\sigma} \mathbf{p}^{\prime \prime}}{I \cdot I I \cdot I I I^{2} \cdot I V^{2}} \\
& -\frac{\left(\mathbf{p}^{\prime}\right)^{2}(I+I I+I I I+I V)}{I(I+I I)(I+I I I)(I+I V)(I I I+I V)(I I I+I I)(I V+I I)} \\
& \left.-\frac{\left(\mathbf{p}^{\prime \prime}\right)^{2}(I+I I+I I I+I V)}{I I(I+I I)(I+I I I)(I+I V)(I I I+I V)(I I I+I I)(I V+I I)}\right) \\
& \times\left\langle\mathbf{p}^{\prime}\left|A^{0}\right| \mathbf{p}^{\prime \prime \prime}\right\rangle\left\langle\mathbf{p}^{\prime \prime \prime}\left|A^{0}\right| \mathbf{p}^{I V}\right\rangle\left\langle\mathbf{p}^{I V}\left|A^{0}\right| \mathbf{p}^{\prime \prime}\right\rangle \\
& +\frac{I \cdot I I}{I I I^{2} \cdot I V^{2}} \sigma^{i} \sigma^{k}\left\langle\mathbf{p}^{\prime}\left|A^{i}\right| \mathbf{p}^{\prime \prime \prime}\right\rangle\left\langle\mathbf{p}^{\prime \prime \prime}\left|A^{0}\right| \mathbf{p}^{I V}\right\rangle\left\langle\mathbf{p}^{I V}\left|A^{k}\right| \mathbf{p}^{\prime \prime}\right\rangle
\end{aligned}
$$




$$
\begin{aligned}
& +\left(-\frac{\boldsymbol{\sigma} \mathbf{p}^{\prime \prime \prime} \sigma^{i}}{I I I^{2}(I+I I I)(I V+I I)}-\frac{\sigma^{i} \boldsymbol{\sigma} \mathbf{p}^{I V}}{I V^{2}(I+I I I)(I V+I I)}\right. \\
& \left.+\frac{\sigma^{i} \boldsymbol{\sigma} \mathbf{p}^{\prime \prime}}{I I \cdot I V^{2}(I+I I I)}+\frac{\boldsymbol{\sigma} \mathbf{p}^{\prime} \sigma^{i}}{I \cdot I I I^{2}(I V+I I)}\right) \\
& \times\left\langle\mathbf{p}^{\prime}\left|A^{0}\right| \mathbf{p}^{\prime \prime \prime}\right\rangle\left\langle\mathbf{p}^{\prime \prime \prime}\left|A^{i}\right| \mathbf{p}^{I V}\right\rangle\left\langle\mathbf{p}^{I V}\left|A^{0}\right| \mathbf{p}^{\prime \prime}\right\rangle-\frac{I \sigma^{i} \sigma^{k}}{I I I^{2}(I V+I I)} \\
& \times\left\langle\mathbf{p}^{\prime}\left|A^{i}\right| \mathbf{p}^{\prime \prime \prime}\right\rangle\left\langle\mathbf{p}^{\prime \prime \prime}\left|A^{k}\right| \mathbf{p}^{I V}\right\rangle\left\langle\mathbf{p}^{I V}\left|A^{0}\right| \mathbf{p}^{\prime \prime}\right\rangle \\
& -\frac{\sigma^{i} \sigma^{k} I I}{I V^{2}(I+I I I)}\left\langle\mathbf{p}^{\prime}\left|A^{0}\right| \mathbf{p}^{\prime \prime \prime}\right\rangle\left\langle\mathbf{p}^{\prime \prime \prime}\left|A^{i}\right| \mathbf{p}^{I V}\right\rangle\left\langle\mathbf{p}^{I V}\left|A^{k}\right| \mathbf{p}^{\prime \prime}\right\rangle \\
& +\left(-\frac{\boldsymbol{\sigma} \mathbf{p}^{\prime} \sigma^{i} I I}{I \cdot I I I^{2} I V^{2}}+\frac{\boldsymbol{\sigma} \mathbf{p}^{\prime \prime \prime} \sigma^{i} I I}{I I I^{2} I V^{2}(I+I I I)}+\frac{\sigma^{I V} \sigma^{i} I I}{I V^{2}(I+I V)(I+I I I)(I I I+I V)}\right. \\
& \left.+\frac{\sigma^{i} \boldsymbol{\sigma} \mathbf{p}^{\prime \prime}}{I I(I+I V)(I+I I I)(I I I+I V)}\right)\left\langle\mathbf{p}^{\prime}\left|A^{0}\right| \mathbf{p}^{\prime \prime \prime}\right\rangle\left\langle\mathbf{p}^{\prime \prime \prime}\left|A^{o}\right| \mathbf{p}^{I V}\right\rangle\left\langle\mathbf{p}^{I V}\left|A^{i}\right| \mathbf{p}^{\prime \prime}\right\rangle \\
& +\left(-\frac{I \sigma^{i} \boldsymbol{\sigma} \mathbf{p}^{\prime \prime}}{I I \cdot I I I^{2} I V^{2}}+\frac{I \sigma^{\prime \prime}}{I I I^{2}(I I I+I I)(I I I+I V)(I V+I I)}+\frac{I \sigma^{i} \boldsymbol{\sigma} \mathbf{p}^{I V}}{I I I^{2} \cdot I V^{2}(I V+I I)}\right. \\
& \left.\left.+\frac{\boldsymbol{\sigma} \mathbf{p}^{\prime} \sigma^{i}}{I(I I I+I I)(I I I+I V)(I V+I I)}\right)\left\langle\mathbf{p}^{\prime}\left|A^{i}\right| \mathbf{p}^{\prime \prime \prime}\right\rangle\left\langle\mathbf{p}^{\prime \prime \prime}\left|A^{0}\right| \mathbf{p}^{I V}\right\rangle\left\langle\mathbf{p}^{I V}\left|A^{0}\right| \mathbf{p}^{\prime \prime}\right\rangle\right\}
\end{aligned}
$$

\section{APPENDIX B. Calculation of matrix elements of QED processes with equations for fermions with spinor wave functions}

1. Electron scattering in Coulomb field: $A^{0}(x)=Z e / 4 \pi|\mathbf{x}|$. The Feynman diagram is presented in Fig.1. Matrix element $S_{f i}$ is

$$
\begin{aligned}
& S_{f i}=-i \int d^{4} x \bar{F}_{0}^{+}\left(x, p_{f}, s_{f}\right) V_{10} A^{0} F_{0}^{+}\left(x, p_{i}, s_{i}\right) \\
& =-\frac{i \delta\left(E_{f}-E_{i}\right)}{(2 \pi)^{2} 2 E_{i}} \bar{U}_{s_{f}} V_{10}\left(p_{f} ; p_{i}\right) A^{0}(q) U_{s_{i}} \\
& =i \frac{Z e^{2}}{\mathbf{q}^{\mathbf{2}}} \frac{\delta\left(E_{f}-E_{i}\right)}{(2 \pi)^{2}} \bar{U}_{s_{f}} \frac{1}{2 E_{i}}\left(E_{i}+m+\frac{1}{E_{i}+m} \boldsymbol{\sigma} \mathbf{p}_{\mathbf{f}} \boldsymbol{\sigma} \mathbf{p}_{\mathbf{i}}\right) U_{s_{i}}
\end{aligned}
$$

where $\mathbf{q}=\mathbf{p}_{\mathbf{f}}-\mathbf{p}_{\mathbf{i}}, A^{0}(\mathbf{q})=4 \pi Z e / \mathbf{q}^{2}$.

In (B.1) for the electron we used function $F_{0}^{+}\left(x, p_{i}, s_{i}\right)$ and the equality of $I I=\left(E_{i}+m\right)^{1 / 2}$ (see 17$), 30$, and 32$)$.

The expression (B.1) coincides with the expression obtained earlier in the Foldy-Wouthuysen representation [13]-[15]. Then, by using conventional methods, we can obtain the differential cross-section of Mott scattering transforming into Rutherford scattering in non-relativistic case (see, for example, Ref. [9]).

2. Scattering of an electron on a Dirac proton (Møller scattering): The Feynman diagram is presented in Fig. 2. 


$$
\begin{aligned}
& S_{f i}=-i \int d^{4} x d^{4} y \bar{F}_{0}^{+}\left(x, p_{f}, s_{f}\right) V_{1}^{\alpha} F_{0}^{+}\left(x, p_{i}, s_{i}\right) D_{f}(x-y) \\
& \times \bar{F}^{+}\left(y, P_{f}, S_{f}\right)\left(-V_{1}\right)_{\alpha} F^{+}\left(y, P_{i}, S_{i}\right)=-\frac{i \delta^{4}\left(P_{f}-P_{i}+p_{f}-p_{i}\right)}{\left(p_{f}-p_{i}\right)^{2}} \\
& \times \frac{2 \pi}{\sqrt{2 p_{i}^{0} 2 p_{f}^{0}} \sqrt{2 P_{i}^{0} 2 P_{f}^{0}}}\left(\bar{U}_{s_{f}} V_{1}^{\alpha}\left(p_{f} ; p_{i}\right) U_{s_{i}}\right)\left(\bar{U}_{S_{f}} V_{1 \alpha}\left(P_{f} ; P_{i}\right) U_{S_{i}}\right) .
\end{aligned}
$$

Above, $D_{f}(x-y)$ is a photon propagator. Matrix element $S_{f i}$ allows determination of Møller cross-section.

3. Compton scattering of electrons: The Feynman diagrams are presented in Fig.3.

We will describe the incident photon with momentum $k^{\mu}$ and polarization $\varepsilon^{\mu}$ by plane wave

$$
A^{\mu}(x, k)=\frac{\varepsilon^{\mu}}{\sqrt{2 k^{0}(2 \pi)^{3}}} e^{-i k x} .
$$

The emitted photon with momentum $k^{\prime \mu}$ and polarization $\varepsilon^{\prime \mu}$ is described by plane wave

$$
A^{\mu}\left(y, k^{\prime}\right)=\frac{\left(\varepsilon^{\prime}\right)^{\mu}}{\sqrt{2\left(k^{\prime}\right)^{0}(2 \pi)^{3}}} e^{i k^{\prime} y} .
$$

The matrix element of the process is

$$
\begin{aligned}
& S_{f i}=-i \bar{U}_{s_{f}}\left\{\int \frac{d^{4} z d^{4} y d^{4} p^{\prime \prime \prime}}{(2 \pi)^{10} \sqrt{2 k^{0} 2\left(k^{\prime}\right)^{0} 2 p_{i}^{0} 2 p_{f}^{0}}}\right. \\
& \times\left(e^{i p_{f} y} V_{1 \mu} \varepsilon^{\prime \mu} e^{i k^{\prime} y} \frac{e^{-i p^{\prime \prime \prime} y}}{\left(p^{\prime \prime \prime}\right)^{2}-m^{2}} e^{i p^{\prime \prime \prime} z} V_{1 \mu} \varepsilon^{\nu} e^{-i k z} e^{i p_{i} z}\right. \\
& \left.+e^{i p_{f} y} V_{1 \mu} \varepsilon^{\mu} e^{-i k y} \frac{e^{-i p^{\prime \prime} y}}{(p)^{2}-m^{2}} e^{i p^{\prime \prime \prime} z} V_{1 \nu} \varepsilon^{\prime \nu} e^{i k^{\prime} z} e^{-i p_{i} z}\right) \\
& +\int d^{4} y \frac{1}{(2 \pi)^{6} \sqrt{2 k^{0} 2\left(k^{\prime}\right)^{0} 2 p_{i}^{0} 2 p_{f}^{0}}}\left(e^{i p_{f} y} V_{2 \mu \nu} \varepsilon^{\prime \mu} e^{i k^{\prime} y} \varepsilon^{\nu} e^{-i k y} e^{-i p_{i} y}\right. \\
& \left.\left.+e^{i p_{f} y} V_{2 \mu \nu} \varepsilon^{\mu} e^{-i k y} \varepsilon^{\prime \nu} e^{i k^{\prime} y} e^{-i p_{i} y}\right)\right\} U_{s_{i}}= \\
& -\frac{i \delta^{4}\left(p_{i}+k-p_{f}-k^{\prime}\right)}{(2 \pi)^{2} \sqrt{2 k^{0} 2\left(k^{\prime}\right)^{0} 2 p_{i}^{0} 2 p_{f}^{0}}}\left(\bar{U}_{s_{f}} M U_{s_{i}}\right),
\end{aligned}
$$

where

$$
\begin{aligned}
& M=V_{1 \mu}\left(p_{f} ; p_{i}+k\right) \varepsilon^{\prime \mu} \frac{1}{\left(p_{i}+k\right)^{2}-m^{2}} V_{1 \mu}\left(p_{i}+k ; p_{i}\right) \varepsilon^{\mu} \\
& +V_{2 \mu \nu}\left(p_{f} ; p_{i}+k ; p_{i}\right) \varepsilon^{\prime \mu} \varepsilon^{\nu} \\
& +V_{1 \mu}\left(p_{f} ; p_{i}-k^{\prime}\right) \varepsilon^{\mu} \frac{1}{\left(p_{i}-k^{\prime}\right)^{2}-m^{2}} V_{1 \mu}\left(p_{i}-k^{\prime} ; p_{i}\right) \varepsilon^{\prime \mu} \\
& +V_{2 \mu \nu}\left(p_{f} ; p_{i}-k^{\prime} ; p_{i}\right) \varepsilon^{\mu} \varepsilon^{\prime \nu} .
\end{aligned}
$$


Here, taking into account the conservation of energy-momentum of $\left(p_{i}+k=p_{f}+k^{\prime}\right)$, operators $V_{1 \mu}, V_{2 \mu \nu}$ are determined from expressions (35), (A.1), taken without fields $A^{\mu}, A^{\mu} A^{\nu}$.

If we select special calibration, in which an initial and final photons are transversely polarized in the laboratory system of reference of $\left(\mathbf{p}_{\mathbf{i}}=0, p_{i}^{0}=m\right.$, $\left.\varepsilon^{0}=\left(\varepsilon^{\prime}\right)^{0}=0, \varepsilon \mathbf{k}=\varepsilon^{\prime} \mathbf{k}^{\prime}=0\right)$ then the expression for $S_{f i}$ is simplified:

$$
\begin{aligned}
& M=e^{2} \sqrt{\frac{2 m+k^{0}-\left(k^{\prime}\right)^{0}}{2 m}} 2\left\{\boldsymbol{\varepsilon}^{\prime} \boldsymbol{\varepsilon}+\frac{1}{2 k^{0}\left(2 m+k^{0}-k^{0}\right)} \boldsymbol{\sigma}\left(\mathbf{k}-\mathbf{k}^{\prime}\right) \boldsymbol{\sigma} \boldsymbol{\varepsilon}^{\prime} \boldsymbol{\sigma} \mathbf{k} \boldsymbol{\sigma} \boldsymbol{\varepsilon}\right. \\
& \left.+\frac{1}{2\left(k^{\prime}\right)^{0}\left(2 m+k^{0}-k^{0 \prime}\right)} \boldsymbol{\sigma}\left(\mathbf{k}-\mathbf{k}^{\prime}\right) \boldsymbol{\sigma} \boldsymbol{\varepsilon} \boldsymbol{\sigma} \mathbf{k}^{\prime} \boldsymbol{\sigma} \boldsymbol{\varepsilon}^{\prime}\right\} .
\end{aligned}
$$

While obtaining the latter expression, we used equalities of

$I \equiv \sqrt{2 m+k^{0}-\left(k^{\prime}\right)^{0}}, I I=\sqrt{2 m}, I I I \equiv \sqrt{2 m+k^{0}}$, or $I I I \equiv \sqrt{2 m-\left(k^{\prime}\right)^{0}}$, $\boldsymbol{\sigma} \mathbf{p}^{\prime}=\boldsymbol{\sigma}\left(\mathbf{k}-\mathbf{k}^{\prime}\right), \boldsymbol{\sigma} \mathbf{p}^{\prime \prime}=0, \boldsymbol{\sigma} \mathbf{p}^{\prime \prime \prime}=\boldsymbol{\sigma} \mathbf{k}$, or $\boldsymbol{\sigma} \mathrm{p}^{\prime \prime \prime}=-\boldsymbol{\sigma} \mathbf{k}^{\prime}$.

Then, by using conventional methods, we can obtain the Klein-Nishina-Tamm formula for the differential cross-section of the Compton scattering.

4. Electron-positron pair annihilation: The process of electron-positron pair annihilation is corresponded by the diagram in Fig. 3 with substitution of $\varepsilon, k \rightarrow \varepsilon_{1},-k_{1}, \varepsilon^{\prime}, k^{\prime} \rightarrow \varepsilon_{2}, k_{2}, p_{i} s_{i} \rightarrow p_{-} s_{-}, p_{f} s_{f} \rightarrow p_{+} s_{+}$. When recording matrix element of $S_{+-}$, for the positron, we used the function of $F_{0}^{+}\left(x, p_{+}, s\right)$ and $I=\left(p_{+}^{0}-m\right)^{1 / 2}$ (see Eqs. 17), 30), and (32).

In analogy with Compton scattering, the matrix element of process $S_{+-}$is

$$
S_{+-}=-\frac{\delta^{4}\left(p_{-}+p_{+}-k_{1}-k_{2}\right)}{(2 \pi)^{2} \sqrt{2 k_{1}^{0} 2 k_{2}^{0} \cdot 2 p_{-}^{0} 2 p_{+}^{0}}} \bar{U}_{s_{+}} M_{1} U_{s_{-}}
$$

where operator $M_{1}$, in terms of its structure, considering the above substitution, coincides with operator $M$ in the expression of $S_{f i}$ for Compton scattering of electrons. The expression of (B.5) allows obtaining the differential crosssection of the electron-positron pair annihilation, which coincides with the cross-section of this process calculated in Dirac representation. 


\section{APPENDIX C. Contributions of Figs. 5(i)-5(1) to the Lamb shift}

- Contribution of Fig.5(i)

$$
\begin{aligned}
& e^{3}\left\{A_{1} B_{1} C_{1}+B_{1} C_{1} \frac{\left(\mathbf{p}^{\prime}\right)^{2}-\boldsymbol{\sigma} \mathbf{p}^{\prime} \boldsymbol{\sigma} \mathbf{k}}{I \cdot I I I}+A_{1} C_{1} \frac{\boldsymbol{\sigma} \mathbf{p}^{\prime} \boldsymbol{\sigma} \mathbf{p}^{\prime \prime}-\boldsymbol{\sigma} \mathbf{p}^{\prime} \boldsymbol{\sigma} \mathbf{k}-\boldsymbol{\sigma} \mathbf{k} \boldsymbol{\sigma} \mathbf{p}^{\prime \prime}+\mathbf{k}^{2}}{I I I^{2}}\right. \\
& +A_{1} B_{1} \frac{\left(\mathbf{p}^{\prime \prime}\right)^{2}-\boldsymbol{\sigma} \mathbf{k} \boldsymbol{\sigma} \mathbf{p}^{\prime \prime}}{I \cdot I I I}+C_{1} \frac{\left(\mathbf{p}^{\prime}\right)^{2}-2 \mathbf{p}^{\prime} \mathbf{k}+\mathbf{k}^{2}}{I \cdot I I I^{3}}\left(\boldsymbol{\sigma} \mathbf{p}^{\prime} \boldsymbol{\sigma} \mathbf{p}^{\prime \prime}-\boldsymbol{\sigma} \mathbf{p}^{\prime} \boldsymbol{\sigma} \mathbf{k}\right) \\
& +B_{1} \frac{\mathbf{k}^{2}}{I^{2} \cdot I I I^{2}} \boldsymbol{\sigma} \mathbf{p}^{\prime} \boldsymbol{\sigma} \mathbf{p}^{\prime \prime}+A_{1} \frac{\left(\mathbf{p}^{\prime \prime}\right)^{2}-2 \mathbf{p}^{\prime \prime} \mathbf{k}+\mathbf{k}^{2}}{I \cdot I I I^{3}}\left(\boldsymbol{\sigma} \mathbf{p}^{\prime} \boldsymbol{\sigma} \mathbf{p}^{\prime \prime}-\boldsymbol{\sigma} \mathbf{k} \boldsymbol{\sigma} \mathbf{p}^{\prime \prime}\right) \\
& +\frac{\mathbf{k}^{4}}{I^{2} \cdot I I I^{2}} \boldsymbol{\sigma} \mathbf{p}^{\prime} \boldsymbol{\sigma} \mathbf{p}^{\prime \prime}+B_{1}\left(\boldsymbol{\sigma} \mathbf{p}^{\prime} \boldsymbol{\sigma} \mathbf{p}^{\prime \prime}-\boldsymbol{\sigma} \mathbf{p}^{\prime} \boldsymbol{\sigma} \mathbf{k}\right) \\
& -B_{1} \frac{I^{2}}{I I I^{2}}\left(-\boldsymbol{\sigma} \mathbf{p}^{\prime} \boldsymbol{\sigma} \mathbf{p}^{\prime \prime}+\boldsymbol{\sigma} \mathbf{k} \boldsymbol{\sigma} \mathbf{p}^{\prime \prime}+\boldsymbol{\sigma} \mathbf{p}^{\prime} \boldsymbol{\sigma} \mathbf{k}+3 \mathbf{k}^{2}+4 \mathbf{p}^{\prime} \mathbf{p}^{\prime \prime}-4 \mathbf{p}^{\prime} \mathbf{k}-4 \mathbf{p}^{\prime \prime} \mathbf{k}\right) \\
& -\frac{3 I I I^{2}}{I^{2}} B_{1} \boldsymbol{\sigma} \mathbf{p}^{\prime} \boldsymbol{\sigma} \mathbf{p}^{\prime \prime}+B_{1}\left(\boldsymbol{\sigma} \mathbf{p}^{\prime} \boldsymbol{\sigma} \mathbf{p}^{\prime \prime}-\boldsymbol{\sigma} \mathbf{k} \boldsymbol{\sigma} \mathbf{p}^{\prime \prime}\right) \\
& +\frac{\left(\mathbf{p}^{\prime \prime}\right)^{2}-2 \mathbf{p}^{\prime \prime} \mathbf{k}+\mathbf{k}^{2}}{I I I^{2}}\left(\left(\mathbf{p}^{\prime}\right)^{2}-\boldsymbol{\sigma} \mathbf{p}^{\prime} \boldsymbol{\sigma} \mathbf{k}\right) \\
& -\frac{3 I^{2}}{I I I^{4}}\left(\left(\mathbf{p}^{\prime}\right)^{2}-2 \mathbf{p}^{\prime} \mathbf{k}+\mathbf{k}^{2}\right)\left(\left(\mathbf{p}^{\prime \prime}\right)^{2}-2 \mathbf{p}^{\prime \prime} \mathbf{k}+\mathbf{k}^{2}\right)-\frac{3 k^{2}}{I^{2}} \boldsymbol{\sigma} \mathbf{p}^{\prime} \boldsymbol{\sigma} \mathbf{p}^{\prime \prime} \\
& \left.+\frac{\left(\mathbf{p}^{\prime}\right)^{2}-2 \mathbf{p}^{\prime} \mathbf{k}+\mathbf{k}^{2}}{I I I^{2}}\left(\left(\mathbf{p}^{\prime \prime}\right)^{2}-\boldsymbol{\sigma} \mathbf{k} \boldsymbol{\sigma} \mathbf{p}^{\prime \prime}\right)\right\} \\
& \times \frac{A^{0}(\mathbf{q})}{\left(\left(k^{0}\right)^{2}-\mathbf{k}^{2}-2\left(\mathbf{p}^{\prime}\right)^{0} k^{0}+2 \mathbf{p}^{\prime} \mathbf{k}\right)\left(\left(k^{0}\right)^{2}-\mathbf{k}^{2}-2\left(\mathbf{p}^{\prime \prime}\right)^{0} k^{0}+2 \mathbf{p}^{\prime \prime} \mathbf{k}\right)}, \\
& A_{1}=I^{2}+I I I^{2}-2 m-\frac{\left(\mathbf{p}^{\prime}\right)^{2}}{I(I+I I I)}-\frac{\left(\mathbf{p}^{\prime}\right)^{2}-2 \mathbf{p}^{\prime} \mathbf{k}+\mathbf{k}^{2}}{I I I(I+I I I)}, \\
& C_{1}=I^{2}+I I I^{2}-2 m-\frac{\left(\mathbf{p}^{\prime \prime}\right)^{2}}{I(I+I I I)}-\frac{\left(\mathbf{p}^{\prime \prime}\right)^{2}-2 \mathbf{p}^{\prime \prime} \mathbf{k}+\mathbf{k}^{2}}{I I I(I+I I I)}, \\
& B_{1}=2 I I I^{2}-2 m-\frac{\left(\mathbf{p}^{\prime}\right)^{2}-2 \mathbf{p}^{\prime} \mathbf{k}+\mathbf{k}^{2}}{2 I I I^{2}}-\frac{\left(\mathbf{p}^{\prime \prime}\right)^{2}-2 \mathbf{p}^{\prime \prime} \mathbf{k}+\mathbf{k}^{2}}{2 I I I^{2}} \text {. }
\end{aligned}
$$

- Contribution of Fig. 5(j)

$$
\begin{aligned}
& e^{3}\left\{D E+D \frac{\boldsymbol{\sigma} \mathbf{p}^{\prime} \boldsymbol{\sigma} \mathbf{p}^{\prime \prime}-\boldsymbol{\sigma} \mathbf{k} \boldsymbol{\sigma} \mathbf{p}^{\prime \prime}}{I \cdot I I I^{3}}-D \frac{\boldsymbol{\sigma} \mathbf{p}^{\prime} \boldsymbol{\sigma} \mathbf{p}^{\prime \prime}-\boldsymbol{\sigma} \mathbf{k} \boldsymbol{\sigma} \mathbf{p}^{\prime \prime}-\boldsymbol{\sigma} \mathbf{p}^{\prime} \boldsymbol{\sigma} \mathbf{k}+\mathbf{k}^{2}}{I I I^{3}(I+I I I)}\right. \\
& -D \frac{\left(\mathbf{p}^{\prime \prime}\right)^{2}-\boldsymbol{\sigma} \mathbf{k} \boldsymbol{\sigma} \mathbf{p}^{\prime \prime}}{2 I \cdot I I I^{3}}+E \frac{\left(\mathbf{p}^{\prime}\right)^{2}-\boldsymbol{\sigma} \mathbf{p}^{\prime} \boldsymbol{\sigma} \mathbf{k}}{I \cdot I I I}+\frac{\mathbf{k}^{2}}{2 I^{2} \cdot I I I^{4}} \boldsymbol{\sigma} \mathbf{p}^{\prime} \boldsymbol{\sigma} \mathbf{p}^{\prime \prime} \\
& -\frac{\left(\mathbf{p}^{\prime}\right)^{2}-2 \mathbf{p}^{\prime} \mathbf{k}+\mathbf{k}^{2}}{I \cdot I I I^{4}(I+I I I)}\left(\boldsymbol{\sigma} \mathbf{p}^{\prime} \boldsymbol{\sigma} \mathbf{p}^{\prime \prime}-\boldsymbol{\sigma} \mathbf{p}^{\prime} \boldsymbol{\sigma} \mathbf{k}\right)
\end{aligned}
$$




$$
\begin{aligned}
& +\frac{\left(\mathbf{p}^{\prime}\right)^{2}-\boldsymbol{\sigma} \mathbf{p}^{\prime} \boldsymbol{\sigma} \mathbf{k}}{I I I^{2}}-\frac{3 I^{2}}{I I I^{4}}\left(\left(\mathbf{p}^{\prime}\right)^{2}-2 \mathbf{p}^{\prime} \mathbf{k}+\mathbf{k}^{2}\right)+\frac{3}{2 I^{2}} \boldsymbol{\sigma} \mathbf{p}^{\prime} \boldsymbol{\sigma} \mathbf{p}^{\prime \prime} \\
& -\frac{\boldsymbol{\sigma} \mathbf{p}^{\prime} \boldsymbol{\sigma} \mathbf{p}^{\prime \prime}-\boldsymbol{\sigma} \mathbf{k} \boldsymbol{\sigma} \mathbf{p}^{\prime \prime}}{2 I I I^{2}}-\frac{\boldsymbol{\sigma} \mathbf{p}^{\prime} \boldsymbol{\sigma} \mathbf{p}^{\prime \prime}-\boldsymbol{\sigma} \mathbf{p}^{\prime} \boldsymbol{\sigma} \mathbf{k}}{2 I I I^{2}} \\
& \left.+\frac{I^{2}}{2 I I I^{4}}\left(-\boldsymbol{\sigma} \mathbf{p}^{\prime} \boldsymbol{\sigma} \mathbf{p}^{\prime \prime}+\boldsymbol{\sigma} \mathbf{k} \boldsymbol{\sigma} \mathbf{p}^{\prime \prime}+\boldsymbol{\sigma} \mathbf{p}^{\prime} \boldsymbol{\sigma} \mathbf{k}+3 \mathbf{k}^{2}+4 \mathbf{p}^{\prime} \mathbf{p}^{\prime \prime}-4 \mathbf{p}^{\prime} \mathbf{k}-4 \mathbf{p}^{\prime \prime} \mathbf{k}\right)\right\} \\
& \times \frac{A^{0}(\mathbf{q})}{\left(k^{0}\right)^{2}-\mathbf{k}^{2}-2\left(\mathbf{p}^{\prime}\right)^{0} k^{0}+2 \mathbf{p}^{\prime} \mathbf{k}} . \\
& D=I I I^{2}+I^{2}-2 m-\frac{\left(\mathbf{p}^{\prime}\right)^{2}}{I \cdot I I I}+\frac{2 \mathbf{p}^{\prime} \mathbf{k}}{I I I(I+I I I)}-\frac{\mathbf{k}^{2}}{I I I(I+I I I)}, \\
& E=-1-\frac{\mathbf{p}^{\prime} \mathbf{k}}{2 I I I^{2}(I+I I I)^{2}}+\frac{\mathbf{k}^{2}}{2 I I I^{3}(I+I I I)}+\frac{\left(\mathbf{p}^{2}\right)^{2}}{2 I I I I)^{2}} \\
& -\frac{\mathbf{p}^{\prime \prime} \mathbf{k}}{I I I^{3}(I+I I I)}-\frac{1}{2 I I I)^{2}} \\
& +\left(\mathbf{p}^{\prime \prime}\right)^{2}\left(\frac{1}{2 I I I^{3}(I+I I I)}-\frac{1}{2 I \cdot I I I(I+I I I)^{2}}\right) .
\end{aligned}
$$

- Contribution of Fig. 5(k)

$$
\begin{aligned}
& e^{3}\left\{D_{1} E_{1}+D_{1} \frac{\boldsymbol{\sigma} \mathbf{p}^{\prime} \boldsymbol{\sigma} \mathbf{p}^{\prime \prime}-\boldsymbol{\sigma} \mathbf{p}^{\prime} \boldsymbol{\sigma} \mathbf{k}}{I \cdot I I I^{3}}-D_{1} \frac{\left(\mathbf{p}^{\prime}\right)^{2}-\boldsymbol{\sigma} \mathbf{p}^{\prime} \boldsymbol{\sigma} \mathbf{k}}{2 I \cdot I I I^{3}}\right. \\
& -D_{1} \frac{\left(\boldsymbol{\sigma} \mathbf{p}^{\prime} \boldsymbol{\sigma} \mathbf{p}^{\prime \prime}-\boldsymbol{\sigma} \mathbf{p}^{\prime} \boldsymbol{\sigma} \mathbf{k}-\boldsymbol{\sigma} \mathbf{k} \boldsymbol{\sigma} \mathbf{p}^{\prime \prime}+\mathbf{k}^{2}\right)}{I I I^{3}(I+I I I)}+E_{1} \frac{\left(\mathbf{p}^{\prime \prime}\right)^{2}-\boldsymbol{\sigma} \mathbf{k} \boldsymbol{\sigma} \mathbf{p}^{\prime \prime}}{I I I \cdot I} \\
& +\frac{\mathbf{k}^{2}}{2 I^{2} \cdot I I I^{4}} \boldsymbol{\sigma} \mathbf{p}^{\prime} \boldsymbol{\sigma} \mathbf{p}^{\prime \prime}-\frac{\left(\mathbf{p}^{\prime \prime}\right)^{2}-2 \mathbf{p}^{\prime \prime} \mathbf{k}+\mathbf{k}^{2}}{I \cdot I I I^{4}(I+I I I)}\left(\boldsymbol{\sigma} \mathbf{p}^{\prime} \boldsymbol{\sigma} \mathbf{p}^{\prime \prime}-\boldsymbol{\sigma} \mathbf{k} \boldsymbol{\sigma} \mathbf{p}^{\prime \prime}\right) \\
& -\frac{\boldsymbol{\sigma} \mathbf{p}^{\prime} \boldsymbol{\sigma} \mathbf{p}^{\prime \prime}-\boldsymbol{\sigma} \mathbf{p}^{\prime} \boldsymbol{\sigma} \mathbf{k}}{2 I I I^{2}}-\frac{3 I^{2}}{I I I^{4}}\left(\left(\mathbf{p}^{\prime \prime}\right)^{2}-2 \mathbf{p}^{\prime \prime} \mathbf{k}+\mathbf{k}^{2}\right) \\
& +\frac{3}{2 I^{2}} \boldsymbol{\sigma} \mathbf{p}^{\prime} \boldsymbol{\sigma} \mathbf{p}^{\prime \prime}+\frac{\left(\mathbf{p}^{\prime \prime}\right)^{2}-\boldsymbol{\sigma} \mathbf{k} \boldsymbol{\sigma} \mathbf{p}^{\prime \prime}}{I I I^{2}}-\frac{\boldsymbol{\sigma} \mathbf{p}^{\prime} \boldsymbol{\sigma} \mathbf{p}^{\prime \prime}-\boldsymbol{\sigma} \mathbf{k} \boldsymbol{\sigma} \mathbf{p}^{\prime \prime}}{2 I I I^{2}} \\
& \left.+\frac{I^{2}}{2 I I I^{4}}\left(-\boldsymbol{\sigma} \mathbf{p}^{\prime} \boldsymbol{\sigma} \mathbf{p}^{\prime \prime}+\boldsymbol{\sigma} \mathbf{k} \boldsymbol{\sigma} \mathbf{p}^{\prime \prime}+\boldsymbol{\sigma} \mathbf{p}^{\prime} \boldsymbol{\sigma} \mathbf{k}+3 \mathbf{k}^{2}+4 \mathbf{p}^{\prime} \mathbf{p}^{\prime \prime}-4 \mathbf{p}^{\prime} \mathbf{k}-4 \mathbf{p}^{\prime \prime} \mathbf{k}\right)\right\} \\
& \times \frac{A^{0}(\mathbf{q})}{\left(k^{0}\right)^{2}-\mathbf{k}^{2}-2\left(\mathbf{p}^{\prime \prime}\right)^{0} k^{0}+2 \mathbf{p}^{\prime \prime} \mathbf{k}}, \\
& D_{1}=I^{2}+I I I^{2}-2 m-\frac{\left(\mathbf{p}^{\prime \prime}\right)^{2}}{I \cdot I I I}+\frac{2 \mathbf{p}^{\prime \prime} \mathbf{k}}{I I I(I+I I I)}-\frac{\mathbf{k}^{2}}{I I I(I+I I I)}, \\
& E_{1}=-1+\frac{\mathbf{k}^{2}}{2 I I I^{3}(I+I I I)}-\frac{\mathbf{k}^{2}}{2 I I I^{2}(I+I I I)^{2}}+\frac{\mathbf{p}^{\prime \prime} \mathbf{k}}{I I I^{2}(I+I I I)^{2}} \\
& -\frac{\mathbf{p}^{\prime} \mathbf{k}}{I I I^{3}(I+I I I)}-\frac{\left(\mathbf{p}^{\prime \prime}\right)^{2}}{2 I I I^{2}(I+I I I)^{2}} \\
& +\left(\mathbf{p}^{\prime}\right)^{2}\left(\frac{1}{2 I I I^{3}(I+I I I)}-\frac{1}{2 I I I \cdot I(I+I I I)^{2}}\right) .
\end{aligned}
$$


- Contribution of Fig. $5(l)$

$$
\begin{aligned}
& e^{3}\left\{\left(\mathbf{p}^{\prime}\right)^{2}\left[\frac{1}{2 I I I^{3}(I+I I I)^{3}}-\frac{1}{2 I \cdot I I I^{3}(I+I I I)^{2}}-\frac{1}{2 I^{2} \cdot I I I(I+I I I)^{3}}\right]\right. \\
& +\left(\mathbf{p}^{\prime \prime}\right)^{2}\left[\frac{1}{2 I I I^{3}(I+I I I)^{3}}-\frac{1}{2 I \cdot I I I^{3}(I+I I I)^{2}}-\frac{1}{2 I^{2} \cdot I I I(I+I I I)^{3}}\right] \\
& +\boldsymbol{\sigma} \mathbf{p}^{\prime} \boldsymbol{\sigma} \mathbf{p}^{\prime \prime}\left[-\frac{1}{I \cdot I I I^{4}(I+I I I)}+\frac{1}{I I I^{4}(I+I I I)^{2}}+\frac{1}{I^{2} \cdot I I I^{4}}\right] \\
& +\boldsymbol{\sigma} \mathbf{k} \boldsymbol{\sigma} \mathbf{p}^{\prime \prime}\left[\frac{1}{2 I \cdot I I I^{3}(I+I I I)^{2}}-\frac{1}{I I I^{4}(I+I I I)^{2}}+\frac{1}{I \cdot I I I^{4}(I+I I I)}\right] \\
& +\boldsymbol{\sigma} \mathbf{p}^{\prime} \boldsymbol{\sigma} \mathbf{k}\left[\frac{1}{2 I \cdot I I I^{3}(I+I I I)^{2}}-\frac{1}{I I I^{4}(I+I I I)^{2}}+\frac{1}{I \cdot I I I^{4}(I+I I I)}\right] \\
& \left.-\mathbf{p}^{\prime} \mathbf{k} \frac{1}{I I I^{3}(I+I I I)^{3}}-\mathbf{p}^{\prime \prime} \mathbf{k} \frac{\mathbf{k}^{2}}{I I I^{3}(I+I I I)^{3}}+\frac{1 I I^{3}(I+I I I)^{3}}{I I I^{4}}\right\} A^{0}(\mathbf{q}) .
\end{aligned}
$$

\section{References}

[1] P. A. M. Dirac, The Principles of Quantum Mechanics (Oxford University Press, 1930).

[2] V. P. Neznamov, Theoret. and Math. Phys. 197:3, 1823 (2018).

[3] V. P. Neznamov and I. I. Safronov, Exp. Theor. Phys. 127:647 (2018), arXiv: 1809.08940 [gr-qc].

[4] V. P. Neznamov, I. I. Safronov and V. E. Shemarulin, Exp. Theor. Phys. 127:684 (2018), arXiv: 1810.01960 [gr-qc].

[5] V. P. Neznamov, I.I. Safronov and V. E. Shemarulin, J. Exp. Theor. Phys. 128: 64 (2019), arXiv: 1904.05791 [gr-qc].

[6] V. P. Neznamov and I. I. Safronov, J. Exp. Theor. Phys. 128: 672 (2019), arXiv: 1907.03579 [physics.gen-ph].

[7] G. Gabrielse, D. Hanneke, T. Kinoshita, M. Noi and B. Odom, Phys. Rev. Lett. 97, 030809 (2006).

[8] W. Heitler, The quantum theory of radiation (At the Clarendon Press, Oxford, 1954).

[9] J. Bjorken and S. Drell, Relativistic quantum mechanics (McGraw-Hill Book Company, 1964).

[10] Ya. B. Zeldovich and V. S. Popov, Sov. Phys. Usp. 14, 673-694 (1972).

[11] P. A. I. Dirac, Lectures on quantum field theory, Belfer Graduate School of Science (Yeshiva University, New York, 1967). 
[12] A. I. Akhiyezer and V. B. Berestetsky, Quantum electrodynamics (Nauka, Moscow, 1969).

[13] V. P. Neznamov, VANT, Ser. "Theoretical and applied physics" 1, 3 (1989).

[14] V. P. Neznamov, VANT, Ser. "Theoretical and applied physics", 1-2, 41 (2004).

[15] V. P. Neznamov, Phys. Part. Nucl. 37, 152 (2006), arXiv: hep-th/0411050.

[16] E.I. Lifshitz and L. P. Pitaevskii, Relativistic quantum theory, part 2 (Nauka, Moscow, 1971).

[17] L. S.Hostler, J. Math. Phys. 26, 1348 (1985).

[18] G-J. Ni, Rel. Grav. Cosmol. 1 123-136 (2004), arXiv: 0308038v1.

[19] G-J. Ni, S. Chen, S. Lou and J. Xu, arXiv: 1007.3051v1.

[20] N. Debergh, J-P. Petit and G. D'Agostini, arXiv: 1809.05046v2. 